\title{
Singularity theory and equivariant bifurcation problems with parameter symmetry
}

\author{
BY JACQUES-ÉLIE FURTER, \\ Department of Mathematics and Statistics, Brunel University, Uxbridge UB8 3PM \\ ANGELA MARIA SITTA, \\ Departamento de Matemática, IBILCE-UNESP, Brazil \\ AND IAN STEWART* \\ Mathematics Institute, University of Warwick, Coventry CV4 $7 A L$.
}

(Received 26 January 1995; revised 30 August 1995)

\section{Introduction}

The study of equivariant bifurcation problems via singularity theory (Golubitsky and Schaeffer [8], Golubitsky, Stewart and Schaeffer [9]) has been mainly concerned with models exhibiting spontaneous symmetry-breaking. The solutions of such bifurcation problems lose symmetry as the parameters vary, but the equations that they satisfy retain the same symmetry throughout.

There is another category of equivariant problems where the bifurcation equations possess less symmetry when some parameters are non-zero; this is called forced or induced symmetry breaking. In [16], Vanderbauwhede, using classical techniques in bifurcation theory, has described some of what might happen in that case. Although many of the results are fairly general they have so far mainly been used for forced symmetry-breaking of a full orbit of a continuous Lie group; for instance in the case of periodic forcing of autonomous systems or, in mechanics, in the case of rigid bodies. See, for example, Chillingworth [2].

In this work we apply techniques from singularity theory to multiparameter bifurcation problems $f(x, \lambda)=0$ with symmetry on both the state variables and on the bifurcation parameters. That is, we consider a map-germ $f: \mathbb{R}^{n} \times \mathbb{R}^{k} \rightarrow \mathbb{R}^{n}$ that satisfies

$$
f(\gamma x, \gamma \lambda)=\gamma f(x, \lambda) \quad \forall \gamma \in \Gamma,
$$

where $\Gamma$ is a compact Lie group acting linearly on $\mathbb{R}^{n}$ and on $\mathbb{R}^{k}$. (We will often omit the word 'germ' and refer to maps, but we work throughout with germs, so all results are valid locally near the origin.)

This means that we study forced symmetry-breaking when some constraints are imposed via an action of the symmetry group $\Gamma$ on the parameters. Let $\Sigma \subset \Gamma$ be the isotropy subgroup of $\lambda$. In terms of spontaneous symmetry-breaking, the full equation is $\Sigma$-equivariant, since when $\bar{\lambda} \in \operatorname{Fix}(\Gamma)$ the map $f(x, \bar{\lambda})$ is $\Gamma$-equivariant in $x$. In the language of singularity theory, without additional conditions, we would consider the recognition problem for $\Gamma$-equivariant problems and unfold them in the $\Sigma$-theory. In our case we do not have a 'full' unfolding in the $\Sigma$-theory because $\Gamma$ remains as a residue of symmetry when we enlarge the space to encompass the

* Research supported in part by a grant from the SERC (now EPSRC). 


\section{JaCques-Élte Furter, Angela Maria Sitta and Ian Stewart}

parameters. In that sense the problem is more constrained than the problems considered in Vanderbauwhede[16] and Chillingworth [2].

In [8] and [9] Golubitsky, Stewart and Schaeffer studied, via singularity theory, one-parameter bifurcation problems with symmetry only on the state variables. Peters [12] classified bifurcation problems with a one-dimensional state variable and two bifurcation parameters, and carried out an extension to multiparameter bifurcations with a symmetry group acting independently on both the state variables and the bifurcation parameters - that is, by a 'diagonal' action $(x, \lambda) \mapsto(\gamma x, \gamma \lambda)$. (It is convenient to use the term 'diagonal' even though the two actions are in general different, to indicate that the state space and the parameter space are invariant under the group action.) Simultaneously, in his Ph.D. thesis, Lari-Lavassani[10] wrote about multiparameter bifurcation problems with symmetry on the state variables, following [9] and some algebraic results introduced by Damon in [3].

In Section 2 we set up the general machinery needed to study $\Gamma$-equivariant multiparameter bifurcation problems via singularity theory, for a diagonal linear action of a compact Lie group $\Gamma$ on the state variables $x$ and on the multiparameter $\lambda$. We define an equivalence relation for such bifurcation problems using changes of coordinates (contact equivalence) preserving the bifurcation structure ( $\lambda$-slices) and the symmetry ( $\Gamma$-action) of the problem. Indeed, two maps $f, g$ are said to be equivalent if there exist $T, X, \Lambda$ such that

$$
g(x, \lambda)=T(x, \lambda) f(X(x, \lambda), \Lambda(\lambda)),
$$

where $T(x, \lambda)$ is an invertible matrix and $(x, \lambda) \mapsto(X(x, \lambda), \Lambda(\lambda))$ is a diffeomorphism germ. Both $T$ and $(X, \Lambda)$ must be symmetry- and orientation-preserving, that is, $T(\gamma x, \gamma \lambda) \gamma=\gamma T(x, \lambda), X(\gamma x, \gamma \lambda)=\gamma X(x, \lambda), \Lambda(\gamma \lambda)=\gamma \Lambda(\lambda)$ and $T(0,0), X_{x}(0,0), \Lambda_{\lambda}(0)$ are in the connected components of their respective identity operators.

We show that this context fits into the general framework introduced by Damon [3]. We can derive the main results, the Finite Determinacy and Unfolding theorems, directly or from their abstract equivalent in [3]. The finite co-dimension of the 'extended tangent space' of such $f$ implies both that $f$ is contact equivalent to a finite segment of its Taylor series expansion (finite determinacy) and that any perturbation of $f$ can be represented in a special perturbation $F$ with $k$ parameters, where $k=\operatorname{cod} f$ is the co-dimension of $f$ (universal unfolding). One can also improve some aspects of the results in a similar spirit to Lari-Lavassani [10]. Observe that the theory introduced here extends to cases when the groups acting on the state and the parameter spaces are different (because this reduces to a suitable action of the direct product of the two groups).

In Section 3 we describe an extended version of the theory. We classify, up to topological co-dimension 1, bifurcation problems with two state variables and two bifurcation parameters that are equivariant for a particular action of the dihedral group $\mathbf{D}_{4}$. In complex coordinates $z, \lambda$, this action is defined by

$$
f(\bar{z}, \lambda)=\overline{f(z, \lambda)} \text { and } f(i \bar{z}, \bar{\lambda})=i \overline{f(z, \lambda)} .
$$

This action is motivated by a mathematical model describing the buckling of a square plate when a pair of forces acts on the four edges of the plate, see Section $5 \cdot 1$. We use the classical framework of singularity theory to find the appropriate tangent spaces and higher order terms relative to our chosen equivalence relation, which 
leads to a list of normal forms. We give a corrected version of the generic normal form derived in Peters[12] and extend the classification to all map-germs of topological co-dimension one.

The main challenge in carrying out the classification for multiparameter problems comes from the calculation of the tangent spaces. They are not in general finitely generated modules over a unique ring, but over a system of rings in the sense of Damon [3]. For $\lambda \in \mathbb{R}$ this is not important because we can first disregard some contributions to the tangent space and consider the remaining 'restricted tangent space' (which is now a finitely generated module). This has finite co-dimension if and only if the total module does. Then we can reintroduce the missing part of the tangent space to compute the final quotient. However, for a multi-dimensional parameter $\lambda$ this approach is no longer possible, because the restricted tangent space is in general of infinite co-dimension. We must therefore make more careful use of the algebraic structure.

In doing so, a different but very natural approach appears: the path formulation. This views the bifurcation equation $f(x, \lambda)=0$ as a multiparameter 'path', parametrized by $\lambda$, through a universal unfolding of $f(x, 0)$. In a sister paper, [4], we analyse that path formulation in an algebraic context, with applications to a more extended classification of the $\mathbf{D}_{4}$-equivariant bifurcation problems from Section 3 . The path formulation was introduced in a geometrical context by Golubitsky and Schaeffer $[7,8]$. They relate bifurcation problems in one state variable without symmetry to $\lambda$-paths in the parameter space of the miniversal unfolding, in the sense of catastrophe theory, of the function germ $x^{k}, k \geqslant 2$. However, at that time the techniques of singularity theory were not powerful enough to handle the full power of the path formulation efficiently - not even in theory. This is why contact equivalence with distinguished parameters was developed in [7]. However, current techniques, strengthened by the use of symbolic algebra software, have made the path formulation a much more feasible method, for example see Mond and Montaldi[11]. This section ends with remarks on the relationship between the classification by Golubitsky and Roberts [6] of $\mathbf{D}_{4}$-equivariant problems with one parameter (upon which the action is trivial) and our classification. We also comment on the use of our classification to tackle gradient $\mathbf{D}_{4}$-equivariant bifurcation problems. Some bifurcation problems, like the buckling of elastic shells in Section 5, have a natural gradient structure, which acts as an additional constraint. Even though contact equivalence does not preserve the set of gradients $\mathscr{E}_{\nabla, \lambda}$, it still induces an equivalence relation on $\mathscr{E}_{\nabla, \lambda}$. Moreover, the perturbation (unfolding) theory extends to the gradient case : see Bridges and Furter [1] for general theoretical results on such questions.

Section 4 deals with the geometrical description of the normal forms of $\mathbf{D}_{\mathbf{4}^{-}}$ equivariant bifurcation problems in terms of their bifurcation diagrams. First, we describe for a general function the type of solutions we can expect (via their isotropy subgroup) with their (linearized) stability. In the multiparameter situation it is important to understand what structure is preserved by contact equivalence. In general, only the relative position of open regions in parameter space, where the structure of the zero-set does not change in any important qualitative manner, is preserved. Usually, specific families of one-dimensional slices have no invariant meaning. However, in our situation the symmetry on parameters implies that the 

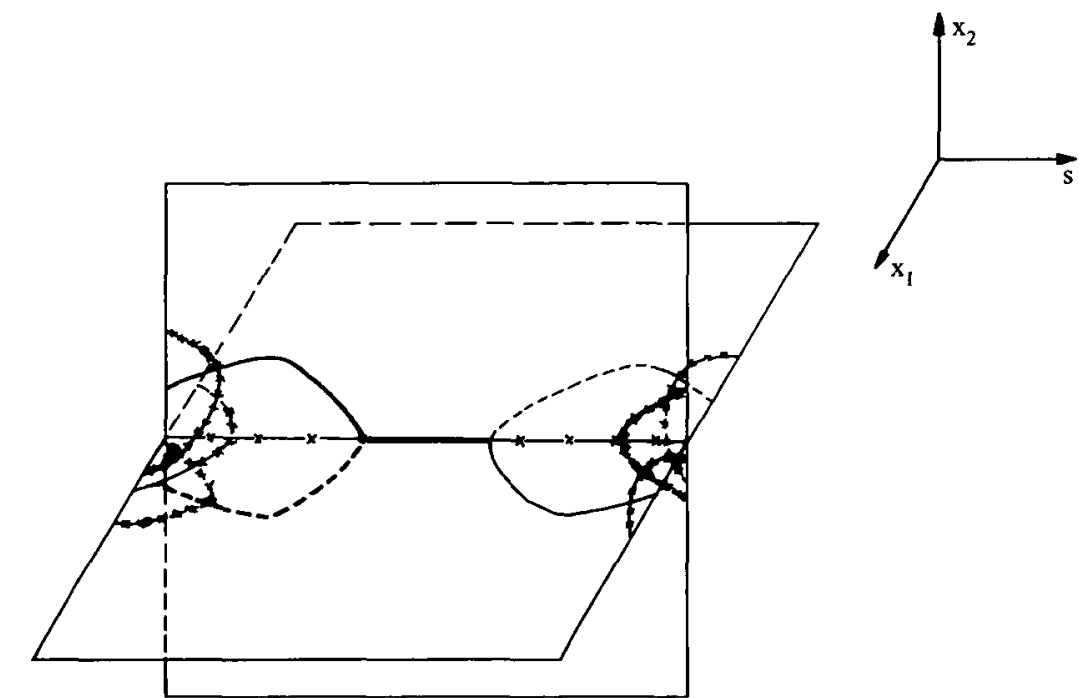

Fig. 1. Bifurcation diagram of the generic normal form when $\epsilon_{5}=1, m>1$. Lines drawn in the form $-x-x-x-$ indicate branches that are always unstable.
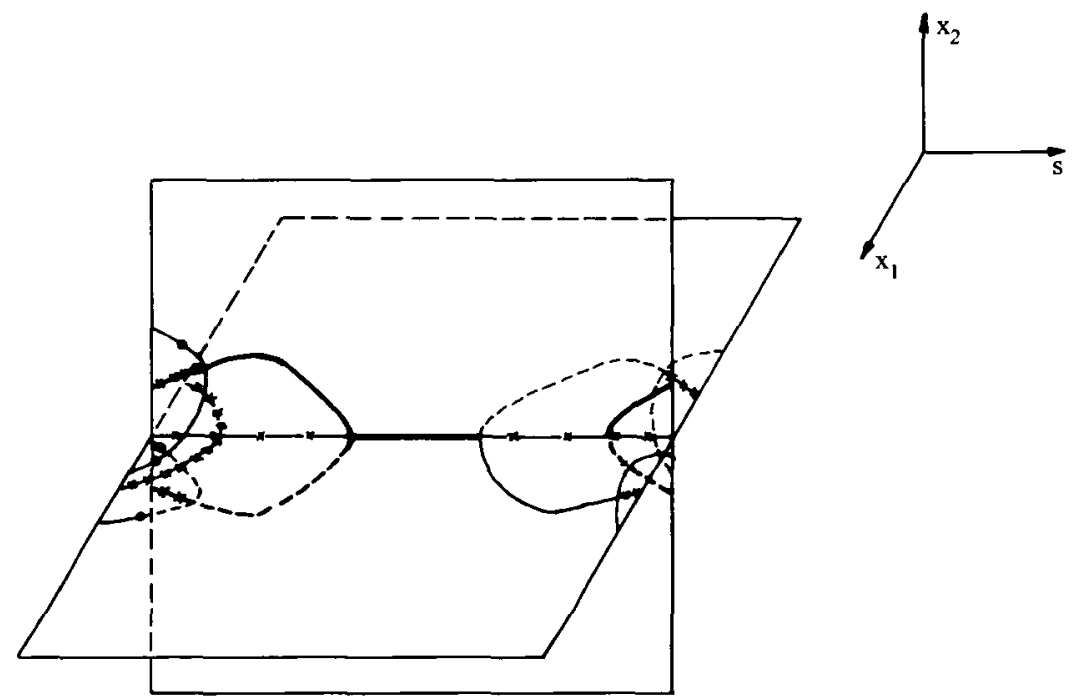

Fig. 2. Bifurcation diagram of the generic normal form when $\epsilon_{5}=-1, m>1$.

axis $\lambda_{2}=0$ is invariant under contact equivalence, so the structure in each half-plane is preserved. Moreover, we can show that the generic normal form $\left(\mathbf{I}_{0}\right.$ in Theorem $3 \cdot 2 \cdot 2)$ is also generic for the stronger contact equivalence respecting $\lambda_{1}$-slices at $\lambda_{2}=$ constant, that is, with $\Lambda\left(\lambda_{1}, \lambda_{2}\right)=\left(\Lambda_{1}\left(\lambda_{1}, \lambda_{2}\right), \Lambda\left(\lambda_{2}\right)\right)$. The proof of that fact is easier in the path formulation, so we refer to the proof in [4]. Hence, in that case, the $\lambda_{2}$ succession of $\lambda_{1}$-slices has a perfectly good invariant meaning.

We analyse the generic normal form in detail. We also draw some schematic diagrams to show the behaviour of the solutions of these bifurcation problems, which show that the trivial solution loses stability to one of the competing single modes. Then we distinguish cases where the solutions without any symmetry (mixed-mode) are stable (Figs 2,4) or unstable (Figs 1,3). There is an exchange of stability between the pure and mixed modes: they are never both stable for the same parameter values. 


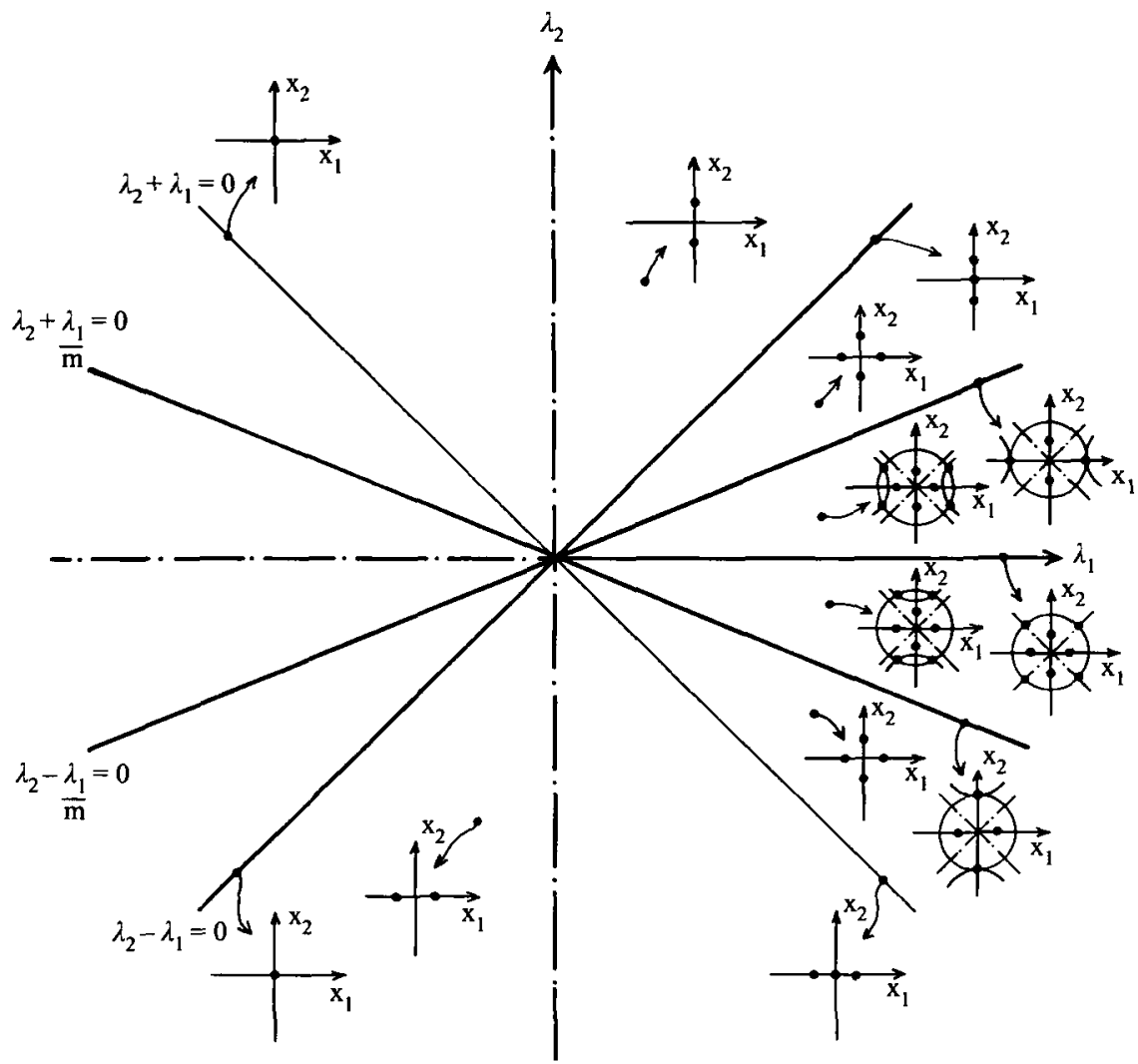

Fig. 3. Solution sets for fixed $\lambda$ for the generic normal form when $\epsilon_{5}=1, m>1$.

In Section 5 we briefly discuss two applications of the theory for $\mathbf{D}_{\mathbf{4}}$-equivariant problems. We consider the buckling of thin elastic shells with a square base under lateral compression, and the bifurcation of steady states of symmetric rings of interacting cells arranged in a square. We concentrate on showing that those two problems can lead to situations where our theory applies. We show that the linearization at the bifurcation has two competing interchangeable modes and the full equations possess the appropriate $D_{4}$-symmetry. We refer to [4] for an analysis of the nonlinear equations.

\section{General theory}

In this section we present a general theory of unfoldings, finite determinacy and the recognition problem for multiparameter equivariant bifurcation problems when a compact Lie group acts diagonally on both the state variables and the bifurcation parameters. Our discussion of this question is based on results of Damon [3] and LariLavassani $[10]$.

\section{2·1. Notation and definitions}

The state variable is $x=\left(x_{1} \ldots x_{n}\right) \in \mathbb{R}^{n}$ and the bifurcation parameter $\lambda=\left(\lambda_{1} \ldots \lambda_{l}\right) \in \mathbb{R}^{l}$. The derivatives are denoted by subscripts, $f_{x}$ for $\partial f / \partial x, \ldots$, and the superscript ${ }^{o}$ denotes the value of any function at the origin, $f^{o}=f(0), f_{x}^{o}=f_{x}(0), \ldots$ 


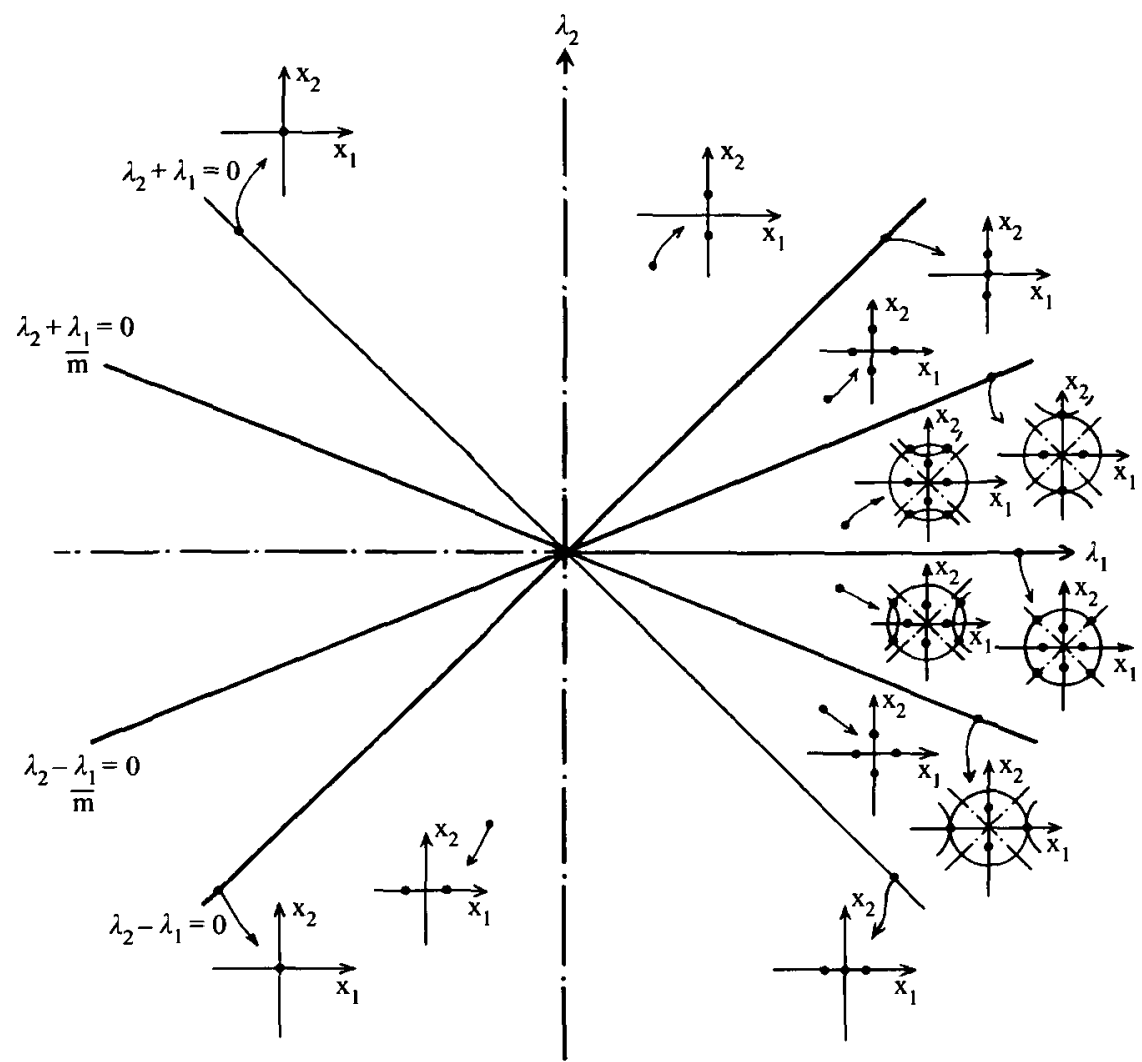

Fig. 4. Solution sets for fixed $\lambda$ for the generic normal form when $\epsilon_{5}=-1, m>1$.

Let $\mathscr{E}_{x}$ denote the ring of smooth germs $f:\left(\mathbb{R}^{n}, 0\right) \rightarrow \mathbb{R}$ and $\mathscr{M}_{x}$ its maximal ideal. For $y \in \mathbb{R}^{m}$ let $\mathscr{E}_{x, y}$ denote the $\mathscr{E}_{x}$-module of smooth germs $g:\left(\mathbb{R}^{n}, 0\right) \rightarrow \mathbb{R}^{m}$, and $\mathscr{M}_{x, y}$ the submodule of germs vanishing at the origin. When $y$ is clear from the context, we denote $\mathscr{E}_{x, y}$ by $\overrightarrow{\mathscr{E}}_{x}$ and $\mathscr{M}_{x, y}$ by $\overrightarrow{\mathscr{M}}_{x}$.

Let GL $(n)$ be the group of all invertible $n \times n$ real matrices and $\mathrm{O}(n)$ the $n$ dimensional orthogonal group. Let $\Gamma$ be a compact Lie group acting on $\mathbb{R}^{m}$ and diagonally on $\mathbb{R}^{n+\ell}$ via orthogonal representations $\rho_{N}: \Gamma \rightarrow \mathrm{O}(N), N=n, l, m$. We denote by $\gamma_{N}$ the action on $\mathbb{R}^{N}$ induced by $\rho_{N}, N=n, l, m$, and identify $\gamma_{N}$ with $\rho_{N}(\gamma)$ for all $\gamma \in \Gamma$. The connected component of the identity map in the subset of GL $(n)$ of the $\Gamma$-equivariant maps is denoted by $\mathscr{L}_{\Gamma}^{o}(n)$. The identity map in GL $(n)$ is denoted by $\mathbf{I}_{n}$.

Let

$$
\mathscr{E}_{(x, \lambda)}^{\Gamma}=\left\{h:\left(\mathbb{R}^{n+\ell}, 0\right) \rightarrow \mathbb{R} \mid h\left(\gamma_{n} x, \gamma_{\ell} \lambda\right)=h(x, \lambda), \quad \forall \gamma \in \Gamma\right\}
$$

be the ring of smooth $\Gamma$-invariant germs and $\mathscr{M}_{(x, \lambda)}^{\Gamma}$ its maximal ideal. There exists a finite set of $\Gamma$-invariant polynomials $\left\{\bar{u}_{i}(x, \lambda)\right\}_{i=1}^{r}$ (Schwarz[13]) such that any element $h \in \mathscr{E}_{(x, \lambda)}$ can be written as the pullback by $\bar{u}=\left(\bar{u}_{1} \ldots \bar{u}_{r}\right)$ of a function of $u=\left(u_{1} \ldots u_{r}\right)$, that is, $\mathscr{E}_{(x, \lambda)}=\bar{u}^{*} \mathscr{E}_{u}$. Similarly, taking

$$
\mathscr{E}_{\lambda}^{\Gamma}=\left\{\eta:\left(\mathbb{R}^{\ell}, 0\right) \rightarrow \mathbb{R} \mid \eta(\gamma, \lambda)=\eta(\lambda), \forall \gamma \in \Gamma\right\}
$$

and $\mathscr{M}_{\lambda}^{\Gamma}$ its maximal ideal, there also exist polynomials $\bar{v}(\lambda)=\left(\bar{v}_{1}(\lambda) \ldots \bar{v}_{t}(\lambda)\right)$ with $\mathscr{E}_{\lambda}^{\Gamma}=\bar{v}^{*} \mathscr{E}_{v}$. 
Let $\overrightarrow{\mathscr{E}}_{(x, \lambda)}^{\Gamma}=\left\{f:\left(\mathbb{R}^{n+\ell}, 0\right) \rightarrow \mathbb{R}^{m} \mid f\left(\gamma_{n} x, \gamma_{\ell} \lambda\right)=\gamma_{m} f(x, \lambda), \forall \gamma \in \Gamma\right\}$ be the $\mathscr{E}_{(x, \lambda)}^{\Gamma}$-module of smooth $\Gamma$-equivariant germs. Then $\overrightarrow{\mathscr{E}}_{(x, \lambda)}^{\Gamma}$ is generated over $\mathscr{E}_{(x, \lambda)}^{\Gamma}$ by a finite set of $\Gamma$-equivariant polynomials maps $\left\{g_{i}\right\}_{i=1}^{\delta}$. Hence, for any $f \in \overrightarrow{\mathscr{E}}_{(x, \lambda)}^{\Gamma}$ there exist some $\left\{h_{j}\right\}_{j=1}^{s} \in \mathscr{E}_{u}$, with

$$
f=\bar{u}^{*}\left(h_{1} g_{1}+\ldots+h_{s} g_{s}\right) .
$$

Thus, we identify $\overrightarrow{\mathscr{E}}_{(x, \lambda)}^{\Gamma}$ with $\bar{u}^{*} \mathscr{E}_{u}^{s}$ (in general that module is not free on $\overrightarrow{\mathscr{E}}_{(x, \lambda)}^{\Gamma}$ ). Similarly, we represent

$$
\overrightarrow{\mathscr{E}}_{\lambda}^{\Gamma}=\left\{\Lambda:\left(\mathbb{R}^{\ell}, 0\right) \rightarrow \mathbb{R}^{\ell} \mid \Lambda\left(\gamma_{\ell} \lambda\right)=\gamma_{\ell} \Lambda(\lambda), \quad \forall \gamma \in \Gamma\right\}
$$

as $\bar{v}^{*} \mathscr{E}_{v}^{\hat{t}}$ for some $\hat{t}$. We denote by $\overrightarrow{\mathscr{M}}_{(x, \lambda)}^{\Gamma}\left(\overrightarrow{\mathscr{M}}_{\lambda}^{\Gamma}\right)$ the submodules of $\overrightarrow{\mathscr{E}}_{(x, \lambda)}^{\Gamma}\left(\overrightarrow{\mathscr{E}}_{\lambda}^{\Gamma}\right)$ of germs vanishing at the origin.

\section{2·2. Contact equivalence}

Next we introduce several distinct equivalence relations, needed to organize the algebra, and their corresponding tangent spaces.

$2 \cdot 2 \cdot 1 . \mathscr{K}_{\lambda}^{\Gamma}$-equivalence. Let

$$
\mathbf{M}_{(x, \lambda)}^{\Gamma}=\left\{T^{\prime}:\left(\mathbb{R}^{n+\ell}, 0\right) \rightarrow M_{m}(\mathbb{R}) \mid T\left(\gamma_{n} x, \gamma_{\ell} \lambda\right) \gamma_{m}=\gamma_{m} T(x, \lambda), \quad \forall \gamma \in \Gamma\right\}
$$

be the $\mathscr{E}_{(x, \lambda)}^{\Gamma}$-module of smooth matrix-valued maps that commute with $\Gamma$.

We also need the following $\mathscr{E}_{(x, \lambda)}^{\Gamma}$-module:

$$
\vec{\Theta}_{(x, \lambda)}^{\Gamma}=\left\{X:\left(\mathbb{R}^{n+\ell}, 0\right) \rightarrow \mathbb{R}^{n} \mid X\left(\gamma_{n} x, \gamma_{\ell} \lambda\right)=\gamma_{n} X(x, \lambda), \quad \forall \gamma \in \Gamma\right\}
$$

and the following $\mathscr{E}_{\lambda}$-module:

$$
\vec{\Theta}_{\lambda}^{\Gamma}=\left\{\Lambda:\left(\mathbb{R}^{\ell}, 0\right) \rightarrow \mathbb{R}^{\ell} \mid \Lambda\left(\gamma_{\ell} \lambda\right)=\gamma_{\ell} \Lambda(\lambda), \quad \forall \gamma \in \Gamma\right\}
$$

with their submodules

$$
\vec{\Theta}_{(\dot{x}, \lambda)}^{\Gamma, o}=\left\{X \in \vec{\Theta}_{(x, \lambda)}^{\Gamma} \mid X^{o}=0\right\}
$$

and

$$
\vec{\Theta}_{\lambda}^{\Gamma, o}=\left\{\Lambda \in \vec{\Theta}_{\lambda}^{\Gamma} \mid \Lambda^{o}=0\right\} .
$$

Appropriate changes of coordinates should not only preserve the zero-set, but also the special role of the bifurcation parameter and the symmetry on both spaces. The contact group $\mathscr{K}_{\lambda}^{\Gamma}$ is thus defined by

$$
\mathscr{K}_{\lambda}^{\Gamma}=\left\{(T, X, \Lambda) \in \mathbf{M}_{(x, \lambda)}^{\Gamma} \times \vec{\Theta}_{(\dot{x}, \lambda)}^{\Gamma, o} \times \vec{\Theta}_{\lambda}^{\Gamma, o} \mid T^{o} \in \mathscr{L}_{\Gamma}^{o}(m), X_{x}^{o} \in \mathscr{L}_{\Gamma}^{o}(n), \Lambda_{\lambda}^{o} \in \mathscr{L}_{\Gamma}^{o}(\ell)\right\}
$$

and it acts in a natural way on $f \in \overrightarrow{\mathscr{E}}_{(x, \lambda)}^{\mathrm{r}}$ by

$$
(T, X, \Lambda) . f(x, \lambda)=T(x, \lambda) f(X(x, \lambda), \Lambda(\lambda)) .
$$

We denote the orbit of this action by $\mathscr{K}_{\lambda}^{\Gamma} . f$. Two elements $f, g \in \overrightarrow{\mathscr{E}}_{(x, \lambda)}^{\Gamma}$ are $\mathscr{K}_{\lambda}^{\Gamma}$ equivalent if they belong to the same $\mathscr{K}_{\lambda}^{\Gamma}$-orbit.

2·2·2. $\mathscr{K}_{\lambda, \text { un }}^{\Gamma}(k)$-equivalence. Let $\beta \in \mathbb{R}^{k}$. We extend in a straightforward manner the definitions of Section $2 \cdot 2 \cdot 1$ to their $\beta$-parametrized versions, $\mathbf{M}_{(x, \lambda, \beta)}^{\Gamma} \overrightarrow{\mathscr{E}}_{(x, \lambda, \beta)}$, $\vec{\Theta}_{(\dot{x}, \lambda, \beta)}^{\Gamma, o}, \vec{\Theta}_{(\dot{\lambda}, \beta)}^{\Gamma, o}$.

Perturbations of any $f \in \overrightarrow{\mathscr{E}}_{(x, \lambda)}^{\Gamma}$ are described by $k$-parameter unfoldings of $f$, which are map-germs $F \in \overrightarrow{\mathscr{E}}_{(x, \lambda, \beta)}^{\Gamma}, \beta=\left(\beta_{1} \ldots \beta_{k}\right)$, such that $F(x, \lambda, 0)=\phi(\xi, \lambda)$. We denote by 
554 Jacques-Élie Furter, Angela Maria Sitta and Ian Stewart

$\mathscr{K}_{\lambda, u n}^{\Gamma}(k)$ the group of $\Gamma$-equivalences for unfoldings with $k$ parameters. It is a natural extension of $\mathscr{K}_{\lambda}^{\Gamma}$ in the following sense:

$\mathscr{K}_{\lambda, u n}^{\Gamma}(k)=\left\{(T, X, \Lambda, \Phi) \in \mathbf{M}_{(x, \lambda, \beta)}^{\Gamma} \times \vec{\Theta}_{(\dot{x}, \lambda, \beta)}^{\Gamma, o} \times \vec{\Theta}_{(\dot{\lambda}, \beta)}^{\Gamma, o} \times \mathscr{M}_{\beta, \beta} \mid(T, X, \Lambda)\right.$

is a $k$ parameter unfolding of an element of $\mathscr{K}_{\lambda}^{\Gamma}$ and $\Phi$ is a diffeomorphism germ $\}$.

The action of $\mathscr{K}_{\lambda, u n}^{\Gamma}(k)$ on $F \in \overrightarrow{\mathscr{E}}_{(x, \lambda, \beta)}^{\Gamma}$ is defined by

$$
(T, X, \Lambda, \Phi) . F(x, \lambda, \beta)=T(x, \lambda, \beta) F(X(x, \lambda, \beta), \Lambda(\lambda, \beta), \Phi(\beta)) .
$$

We say that $F, G \in \overrightarrow{\mathscr{E}}_{(x, \lambda, \beta)}^{\Gamma}$ are $\mathscr{K}_{\lambda, u n}^{\Gamma}(k)$-equivalent if they belong to the same $\mathscr{K}_{\lambda, u n}^{\Gamma}(k)$-orbit.

For later purposes, we distinguish two subgroups which together generate $\mathscr{K}_{\lambda, u n}^{\Gamma}(k)$. The first, denoted by $\mathscr{K}_{u n}^{\Gamma}(k)$, is given by $\Phi=\mathbf{I}_{\beta}$; for the second, $\mathscr{K}_{e q}^{\Gamma}(k)$, the function $(T, X, \Lambda)$ is an unfolding of the identity of $\mathscr{K}_{\lambda}^{\Gamma}$.

$2 \cdot 2 \cdot 3$. Tangent spaces. Associated with $\mathscr{K}_{\lambda}^{\Gamma}$ we can define several different tangent spaces to $f$. The fundamental ones we need in the rest of this paper are the following.

Let $f \in \overrightarrow{\mathscr{E}}_{(x, \lambda)}$. Then the extended tangent space of $f$ is

$$
\mathscr{T}_{e}^{\Gamma}(f)=\left\{T f+f_{x} X+f_{\lambda} \Lambda \mid T \in \mathbf{M}_{(x, \lambda)}^{\Gamma}, X \in \vec{\Theta}_{(x, \lambda)}^{\Gamma}, \Lambda \in \vec{\Theta}_{\lambda}^{\Gamma}\right\} .
$$

Observe that this has only the structure of a $\mathscr{E}_{l} \Gamma$-module.

The extended normal space to $f$ is defined by

$$
\mathcal{N}_{e}^{\Gamma}(f)=\overrightarrow{\mathscr{E}}_{(x, \lambda)}^{\Gamma} / \mathscr{T}_{e}^{\Gamma}(f)
$$

and the $\Gamma$-co-dimension of $f \in \overrightarrow{\mathscr{E}}_{(x, \lambda)}$ is

$$
\operatorname{cod}^{\Gamma}(f)=\operatorname{dim}_{\mathbb{R}} \mathcal{N}_{e}^{\Gamma}(f) .
$$

To simplify some algebraic manipulations it is also useful to introduce the following more restrictive equivalence which brings in tangent spaces that are $\mathscr{E}_{(x, \lambda)^{-}}$ modules. We say that two germs $f, g \in \overrightarrow{\mathscr{M}}_{(x, \lambda)}^{r}$ are strongly $\mathscr{K}_{\lambda}^{\Gamma}$-equivalent if they are $\mathscr{K}_{\lambda}^{\Gamma}$-equivalent with $\Lambda(\lambda)=\lambda$. For our purposes the appropriate tangent space to $f$ associated with strong equivalence is

$$
\mathscr{R} \mathscr{T}^{\Gamma}(f)=\left\{T f+f_{x} X \mid T \in \mathbf{M}_{(x, \lambda)}^{\Gamma}, X \in \vec{\Theta}_{(\dot{x}, \lambda)}^{\Gamma, o}\right\} .
$$

Observe that $\mathscr{R T}^{\Gamma}(f)$ is a finitely generated $\mathscr{E}_{(x, \lambda)}^{\Gamma}$-module. When $\lambda \in \mathbb{R}$, its use is fundamental because the finite co-dimension of $f$ is equivalent to the finite codimension of $\mathscr{R} \mathscr{T}^{\Gamma}(f)$. This is not the case with a multidimensional $\lambda$; nevertheless, the $\mathscr{E}_{(x, \lambda)}^{\Gamma}$-module structure makes $\mathscr{R} \mathscr{T}^{\Gamma}(f)$ of some use in explicit computations. For the recognition problem (Section $2 \cdot 5 \cdot 2$ ) we will encounter yet another type of tangent space associated to the subgroup of unipotent equivalence.

\subsection{Unfolding theory}

Let $F^{\prime} \in \overrightarrow{\mathscr{E}} \Gamma_{(x, \lambda, \beta)}$ and $\left.G \in \overrightarrow{\mathscr{E}} \Gamma_{(x, \lambda, \alpha)}\right)$ be two unfoldings of $f \in \overrightarrow{\mathscr{E}}_{(x, \lambda)}$ with $k$ and $r$ parameters respectively. We say that $G$ maps into or factors through $F$ if there exist $T \in \mathbf{M}_{(x, \lambda, \alpha)}^{\Gamma}, X \in \vec{\Theta}_{(x, \lambda, \alpha)}^{\Gamma}, \Lambda \in \vec{\Theta}_{(\lambda, \alpha)}^{\Gamma}$ and $A:\left(\mathbb{R}^{r}, 0\right) \rightarrow\left(\mathbb{R}^{k}, 0\right)$ satisfying $T(x, \lambda, 0)=\mathbf{I}_{m}$, $X(x, \lambda, 0)=x$ and $\Lambda(\lambda, 0)=\lambda$ such that

$$
G(x, \lambda, \alpha)=T(x, \lambda, \alpha) F(X(x, \lambda, \alpha), \Lambda(\lambda, \alpha), A(\alpha)) .
$$


The unfolding $F$ is versal if any unfolding $G$ of $f$ maps into $F$. If $F$ is versal and has minimal number of parameters, it is called a miniversal unfolding of $f$. The miniversal unfoldings of $f$ are unique up to $\mathscr{K}_{\lambda, u n}^{\Gamma}(k)$-equivalence (cf. Theorem 2.3.3).

From Damon's general theory ([3], of. Section $2 \cdot 6$ here), or directly (Sitta [14]), we have the usual results from singularity theory, as follows.

Theorem 2:3·1. (Unfolding Theorem). Let $f \in \overrightarrow{\mathscr{E}}_{(x, \lambda)}^{\Gamma}$ and $F \in \overrightarrow{\mathscr{E}}_{(x, \lambda, \alpha)}$ be an unfolding of $f$ with $k$ parameters, $\alpha=\left(\alpha_{1} \ldots \alpha_{k}\right)$. Then

1. The unfolding $F$ is versal if and only if

$$
\overrightarrow{\mathscr{E}}_{(x, \lambda)}^{\Gamma}=\mathscr{T}_{e}^{\Gamma}(f)+\mathbb{R} \cdot\left\langle F_{\alpha_{1}}(\cdot, \cdot, 0) \ldots F_{\alpha_{k}}(\cdot, \cdot, 0)\right\rangle .
$$

2. Two versal unfoldings of a germ in $\overrightarrow{\mathscr{E}}_{(x, \lambda)}^{\Gamma}$ are equivalent as unfoldings if and only if they have the same number of unfolding parameters.

CoRolLaRY 232. Let $g \in \overrightarrow{\mathscr{E}}_{(x, \lambda)}^{r}$ be of finite co-dimension and let $W \subset \overrightarrow{\mathscr{E}}_{(x, \lambda)}^{r}$ be a complement of $\mathcal{N}_{e}^{\Gamma}(g)$ as a vector space, $\overrightarrow{\mathscr{E}}_{(x, \lambda)}^{\Gamma}=\mathscr{T}_{e}^{\Gamma}(g) \oplus W$. Let $\left\{p_{i}\right\}_{i-1}^{k}$ be a basis for $W$. Then

$$
G(x, \lambda, \alpha)=g(x, \lambda)+\sum_{j=1}^{k} \alpha_{j} p_{j}(x, \lambda)
$$

is a miniversal unfolding of $g$.

From this corollary we get:

THEOREM 2:33. (Uniqueness of Miniversal Unfolding). If $f$ and $g \in \overrightarrow{\mathscr{E}}_{(x, \lambda)}^{\Gamma}$ are two $\mathscr{K}_{\lambda}^{\Gamma}$. equivalent germs of finite co-dimension and $F$ and $G \in \overrightarrow{\mathscr{E}}_{(x, \lambda, \alpha)}$, with $\alpha=\left(\alpha_{1} \ldots \alpha_{k}\right)$, are two miniversal unfoldings of $f$ and $g$ respectively, then $F$ and $G$ are $\mathscr{K}_{\lambda, u n}^{\Gamma}(k)$-equivalent.

In this case we say that $F, G$ are universal unfoldings.

\section{2·4. Determinacy}

For any mapping $f$ we let $j^{k}(f)$ denote the Taylor polynomial of order $k$, or $k$-jet, of $f$. A germ $f \in \overrightarrow{\mathscr{E}}_{(x, \lambda)}^{\Gamma}$ is $k-\mathscr{K}_{\lambda}^{\Gamma}$-determined if every germ $g \in \overrightarrow{\mathscr{E}}_{(x, \lambda)}^{\Gamma}$ with $j^{k}(g)=j^{k}(f)$ is $\mathscr{K}_{\lambda}^{\Gamma}$-equivalent to $f$. A germ is finitely $\mathscr{K}_{\lambda}^{\Gamma}$-determined if it is $k$ - $\mathscr{L}_{\lambda}^{\Gamma}$-determined for some integer $k$.

As usual, there is a close relationship between being finitely determined and being of finite co-dimension. The first theorem follows from the general theory.

Theorem 2:4·1. (Finite Determinacy). Let $f \in \overrightarrow{\mathscr{E}}_{(x, \lambda)}^{\Gamma}$. Then $f$ is finitely $\mathscr{K}_{\lambda}^{\Gamma}$-determined if and only if $\operatorname{cod}^{\Gamma}(f)$ is finite.

The following theorem gives a necessary condition for a germ $p \in \overrightarrow{\mathscr{E}}_{(x, \lambda)}$ to be in the set of higher order terms of $f$; that is, those terms that can be removed (by an equivalence) from the Taylor expansion of $f$. See theorem $2 \cdot 2$ of Gaffney [5].

Theorem 2-4.2. Let $f \in \overrightarrow{\mathscr{E}}_{(x, \lambda)}^{\Gamma}$ and let $p$ be any germ in $\overrightarrow{\mathscr{E}}_{(x, \lambda)}$. Then

1. If $\mathscr{R} \mathscr{T}^{\mathrm{\Gamma}}(f+t p)=\mathscr{R} \mathscr{T}^{\Gamma}(f), \forall t \in[0,1]$, then $f+p$ is strongly $\mathscr{K}_{\lambda}^{\Gamma}$-equivalent to $f$.

2. If $\operatorname{cod}^{\Gamma}(f)$ is finite and $\mathscr{T}_{e}^{\Gamma}(f+t p)=\mathscr{T}_{e}^{\Gamma}(f), \forall t \in[0,1]$, then $f+p$ is $\mathscr{K}_{\lambda}^{\Gamma}$-equivalent to $f$.

Proof. See Sitta [14]. 


\section{Jacques-Élie Furter, Angela Maria Sitta and Ian Stewart}

\subsection{The recognition problem}

The recognition problem is the determination of conditions on jets for a germ $g \in \overrightarrow{\mathscr{E}}_{(x, \lambda)}^{\Gamma}$ to be $\mathscr{K}_{\lambda}^{r}$-equivalent to a given normal form. To solve a particular recognition problem means to characterize explicitly the $\mathscr{K}_{\lambda}^{\Gamma}$-equivalence class in terms of a finite number of polynomial equalities and inequalities to be satisfied by the Taylor coefficients of the elements of the class.

2.5.1. Intrinsic submodules and higher order terms. Let $\Phi=(T, X, \Lambda) \in \mathscr{K}_{\lambda}^{\Gamma}$ and consider the mapping $f \mapsto \Phi(f)=T$. $f \circ(X, \Lambda)$. Say that a submodule $M \subset \overrightarrow{\mathscr{E}}_{(x, \lambda)}^{\Gamma}$ is intrinsic if $\Phi(f) \in M$ for all $f \in M$ and for all $\Phi \in \mathscr{K}_{\lambda}^{\Gamma}$. Let $V \subset \overrightarrow{\mathscr{E}}_{(x, \lambda)}$. Then the intrinsic part of $V$, denoted by Intr $V$, is defined to be the largest intrinsic submodule of $\overrightarrow{\mathscr{E}}_{(x, \lambda)}$ contained in $V$.

Let $f \in \overrightarrow{\mathscr{E}}_{(x, \lambda)}^{\Gamma}$. The 'perturbation term' $p \in \overrightarrow{\mathscr{E}}_{(x, \lambda)}^{\Gamma}$ is of higher order with respect to $f$ if $g+p$ is $\mathscr{K}_{\lambda}{ }_{\lambda}$-equivalent to $f$ for every $g$ that is $\mathscr{K}_{\lambda}{ }_{\lambda}$-equivalent to $f$. By definition, such a perturbation cannot enter into a solution of recognition problem for $f$. We denote by $\mathscr{P}(f)$ the set of all higher order terms of $f$, that is,

$$
\mathscr{P}(f)=\left\{p \in \overrightarrow{\mathscr{E}}_{(x, \lambda)}^{\Gamma} \mid g+p \sim f, \forall g \sim f\right\}
$$

where $\sim$ denotes $\mathscr{K}_{\lambda}^{\Gamma}$-equivalence. With the same proof as for proposition $7 \cdot 5$ of [9], we have the following:

Proposition 2:5・1. For each $f \in \overrightarrow{\mathscr{E}}_{(x, \lambda)}$, the set $\mathscr{P}(f)$ is an intrinsic submodule of $\overrightarrow{\mathscr{E}}_{(x, \lambda)}^{\Gamma}$.

$2 \cdot 5 \cdot 2$. Unipotent $\mathscr{K}_{\lambda}^{\Gamma}$-equivalences. Another useful subgroup of $\mathscr{K}_{\lambda}^{\Gamma}$ is the group of (restricted) unipotent equivalences. The kernel of the projection map $\pi$ sending $(T, X, \Lambda) \in \mathscr{K}_{\lambda}^{\Gamma}$ onto $\left(T^{o}, X_{x}^{o}, \Lambda_{\lambda}^{o}\right)$ is

$$
\mathscr{U}_{\lambda}^{\Gamma}=\left\{(T, X, \Lambda) \in \mathscr{K}_{\lambda}^{\Gamma} \mid T^{o}=\mathbf{I}_{m}, X_{x}^{o}=\mathbf{I}_{n}, \Lambda_{\lambda}^{o}=\mathbf{I}_{\ell}\right\} .
$$

It is a normal subgroup of $\mathscr{K}_{\lambda}^{\Gamma}$ consisting of unipotent diffeomorphisms and it is called the subgroup of unipotent $\Gamma$-equivalences. Its associated tangent space to $f \in$ $\overrightarrow{\mathscr{E}} \Gamma_{(x, \lambda)}$ is

$$
\mathscr{T} \mathscr{U}^{\Gamma}(f)=\left\{T f+f_{x} X+f_{\lambda} \Lambda \mid T \in \mathbf{M}_{(x, \lambda)}^{\Gamma}, X \in \vec{\Theta}_{(\dot{x}, \lambda)}^{\Gamma, o}, \Lambda \in \vec{\Theta}_{\lambda}^{\Gamma, o}, T^{o}=0, X_{x}^{o}=0, \Lambda_{\lambda}^{o}=0\right\} .
$$

For computational reasons we also define a restricted unipotent tangent space at $f$, given by

$$
\mathscr{R} \mathscr{T} \mathscr{U}^{\Gamma}(f)=\left\{T f+f_{x} X \mid\left(T, X, \mathrm{I}_{\ell}\right) \in T \in \mathbf{M}_{(x, \lambda)}^{\Gamma}, X \in \vec{\Theta}_{(\dot{x}, \lambda)}^{\Gamma, o}, T^{o}=0, X_{x}^{o}=0\right\} .
$$

By theorem 1.17 of Gaffney[5] we have:

Proposition 2:5*3. Let $f \in \overrightarrow{\mathscr{E}}_{(x, \lambda)}^{\Gamma}$ be of finite $\Gamma$-co-dimension. Then $\mathscr{P}(f) \supset \operatorname{Intr} \mathscr{T} \mathscr{U}^{\Gamma}(f)$.

CoRollary 2:5*4. Let $p \in \operatorname{Intr} \mathscr{T} \mathscr{U}^{\Gamma}(f)$, then $f+p$ is $\mathscr{K}_{\lambda}^{\Gamma}$-equivalent to $f$.

\section{Geometric subgroups and proofs}

2.6.1. Definitions. We recall some basic definitions and some results due to Damon [3] which are necessary to establish the Unfolding and Finite Determinacy Theorems.

Let $y \in \mathbb{R}^{n}$ and $y^{\prime} \in \mathbb{R}^{m}$. A DA-algebra A (differentiable algebra) consists of an $\mathbb{R}$ - 
algebra $A$ and a surjective algebra homomorphism $\psi: \mathscr{E}_{y} \rightarrow A$. These algebras are local rings with maximal ideals $\mathscr{M}_{A}$. If $\phi: \mathscr{E}_{y^{\prime}} \rightarrow B$ defines another DA-algebra, then a homomorphism of DA-algebras $\alpha: A \rightarrow B$ is an algebra homomorphism which lifts to $\tilde{\alpha}: \mathscr{E}_{y} \rightarrow \mathscr{E}_{y^{\prime}}$ with $\tilde{\alpha}=g^{*}$ for some $g:\left(\mathbb{R}^{m}, 0\right) \rightarrow\left(\mathbb{R}^{n}, 0\right)$ and $\alpha \circ \psi=\phi \circ \tilde{\alpha}$. For example, $\mathscr{E}_{(x, \lambda)}^{\Gamma}$ and $\mathscr{E}_{\lambda}^{\Gamma}$ are DA-algebras. Moreover, $\mathscr{M}_{(x, \lambda)}^{\Gamma}$ and $\mathscr{M}_{\lambda}^{\Gamma}$ are Jacobson ideals (that is, $1+f$ is always invertible for $f \in \mathscr{M}$ ).

Let $(\mathscr{D},<)$ be a finite partially ordered set of indices. A system of DA-algebras (associated to) $\mathscr{D}$ consists of a set of DA-algebras $\left\{R_{\alpha}\right\}_{\alpha \in \mathscr{D}}$ together with homomorphisms of DA-algebras $\phi_{\alpha \beta}: R_{\alpha} \rightarrow R_{\beta}$ defined for $\alpha \leqslant \beta$ so that $\phi_{\beta \gamma} \circ \phi_{\alpha \beta}=\phi_{\alpha \gamma}$, for $\alpha \leqslant \beta \leqslant \gamma$, and $\phi_{\alpha \alpha}=i d$. A system of ideals $\left\{\mathscr{I}_{\alpha}\right\}$ of $\left\{R_{\alpha}\right\}$ consists of ideals $\mathscr{I}_{\alpha} \subset R_{\alpha}$ so that $\phi_{\alpha \beta}\left(\mathscr{I}_{\alpha}\right) \subseteq \mathscr{I}_{\beta}$ for $\alpha \leqslant \beta$. Then, $\left\{\left(R_{\alpha}, \mathscr{I}_{\alpha}\right)\right\}$ denotes a system of DA-algebras and ideals. For example, $\left\{\mathscr{E}_{\lambda}^{\Gamma}, \mathscr{E}_{(x, \lambda)}^{\Gamma}\right\}$ is a system of DA-algebras. More generally, $\left\{\mathscr{E}_{\beta}, \mathscr{E}_{(x, \beta)}^{\Gamma}, \mathscr{E}_{(x, \lambda, \beta)}^{\Gamma}\right\}$, is also a system of DA-algebras.

To specify in this system the maximal ideals we define the system of rings and ideals

$$
\left\{R_{(x, \lambda, \beta)}\right\}=\left\{\left(\mathscr{E}_{\beta}, \mathscr{M}_{\beta}\right),\left(\mathscr{E}_{(\lambda, \beta)}^{\Gamma}, \mathscr{M}_{(\lambda, \beta)}^{\Gamma}\right),\left(\mathscr{E}_{(x, \lambda, \beta)}^{\Gamma}, \mathscr{M}_{(x, \lambda, \beta)}^{\Gamma}\right)\right\}
$$

An $\left.\left\{R_{(x, \lambda, \beta}\right)\right\}$-module $M$ consists of a direct $\operatorname{sum} M_{1} \oplus M_{2} \oplus M_{3}$ such that $M_{1}$ is an $\mathscr{E}_{l}^{\Gamma}$ module, $M_{2}$ is an $\mathscr{E}_{(x, \lambda)}^{\Gamma}$-module and $M_{3}$ is an $\mathscr{E}_{(x, \lambda \rho)}^{\Gamma}$-module. The module $M$ is said to be finitely generated if each $M_{i}$ is a finitely generated module over the corresponding ring, $1 \leqslant i \leqslant 3$. An $\left\{R_{(x, \lambda, \beta)}\right\}$-module homomorphism $\psi: M \rightarrow N$ consists of a sum of homomorphisms $\psi_{j i}: M_{j} \rightarrow N_{i}$, for $1 \leqslant j, i \leqslant 3$, which are homomorphisms over the appropriate connecting homomorphisms $\pi_{j i}^{*}$. We say that $N$ is an $\left\{R_{(x, \lambda, \beta)}\right\}$-submodule of $M$ if $N=N_{1} \oplus N_{2} \oplus N_{3}$, where $N_{i}$ is a submodule of $M_{i}$ for all $1 \leqslant i \leqslant 3$. If $\left\{\mathscr{I}_{(x, \lambda, \beta)}\right\}=\left\{\mathscr{I}_{\beta}, \mathscr{I}_{(\lambda, \beta)}, \mathscr{I}_{(x, \lambda, \beta)}\right\}$ is a system of ideals of $\left\{R_{(x, \lambda, \beta)}\right\}$, we define

$$
\left\{\mathscr{I}_{(x, \lambda, \beta)}\right\} \cdot M=\mathscr{I}_{\beta} \cdot M_{1} \oplus \mathscr{I}_{(\lambda, \beta)} \cdot M_{2} \oplus \mathscr{I}_{(x, \lambda, \beta)} \cdot M_{3}
$$

and it is an $\left\{R_{(x, \lambda, \beta)}\right\}$-submodule of $M$.

Similar definitions can be made for the system $\left\{R_{(x, \lambda)}\right\}$ by setting $\beta=0$.

Note that each ring in $\left\{R_{(x, \lambda, \beta)}\right\}$ is a $\mathscr{E}_{\beta^{-}}$-algebra. We say that $\left\{R_{(x, \lambda, \beta)}\right\}$ is an adequately ordered system of DA-algebras over $\mathscr{E}_{\beta}$ if each connecting homomorphism is an $\mathscr{E}_{\beta}$-algebra homomorphism and each ring has precisely one predecessor.

2.6.2. Geometric subgroups. In this section we recall the general framework for singularity theory developed by Damon in [3] and show how we can apply this framework in our context. Let $\mathscr{G}$ be a subgroup of the contact equivalence group $\mathscr{K}$ acting on a linear subspace $\mathscr{F}$ of $\overrightarrow{\mathscr{E}}_{x}$. We assume for each $\mathbb{R}^{q}$, that we are given a group of unfoldings $\mathscr{G}_{u n}(q)$ (a subgroup of $\mathscr{K}_{u n}(q)$ ) acting on a subspace $\mathscr{F}_{u n}(q) \subset \overrightarrow{\mathscr{E}}_{(x, \beta)}$ of unfoldings with $q$ parameters, $\beta=\left(\beta_{1} \ldots \beta_{q}\right)$, of germs in $\overrightarrow{\mathscr{E}}_{x}$. For $q=0$, we will have exactly $\mathscr{G}$ and $\mathscr{F}$.

A group $\mathscr{G}$ acting on $\mathscr{F} \subset \overrightarrow{\mathscr{E}}_{x}$ (together with the associated unfolding group $\mathscr{G}_{u n}(q)$ acting on $\left.\mathscr{F}_{u n}(q) \subset \overrightarrow{\mathscr{E}}_{(x, \beta)}\right)$ will be called a geometric subgroup of $\mathscr{K}$ if it satisfies the following four conditions:

- Naturality;

- Tangent Space;

- Exponential Map;

- Filtration Preservation.

We explain these four conditions in turn. 


\section{Jacques-Élie Furter, Angela Maria Sitta and Ian Stewart}

Naturality. The group and space of unfoldings are natural with respect to pullback. We can consider elements in $\mathscr{G}_{u n}(q+1)$ and $\mathscr{F}_{u n}(q+1)$ as unfoldings of elements in $\mathscr{G}_{u n}(q)$ and $\mathscr{F}_{u n}(q)$ via the pullback by the immersion $\mathbb{R}^{q} \hookrightarrow \mathbb{R}^{q+1}$. Thus we can compute the extended spaces $\mathscr{T}_{e} \mathscr{G}_{u n}(q)$ and $\mathscr{T}_{e} \mathscr{F}_{u n}(q)$.

Tangent space. This describes the algebraic structure of the tangent spaces and extended tangent spaces and their relations. It takes the form of three requirements, as follows.

First, there is a collection of $D A$-subalgebras $\left\{R_{x}\right\}$ of $\mathscr{F}$ such that for each $\mathbb{R}^{q}$ with local coordinates $\beta$ the modules $\mathscr{T}_{e} \mathscr{G}_{u n}(q)$ and $\mathscr{T}_{e} \mathscr{F}_{u n}(q)$ are finitely generated over $\left\{R_{(x, \beta)}\right\}$, with $\mathscr{T} \mathscr{G}_{u n}(q)$ and $\mathscr{T} \mathscr{F}_{u n}(q)$ finitely generated $\left\{R_{(x, \beta)}\right\}$-submodules and, containing (via naturality) $\mathscr{T}_{e} G$ and $\mathscr{T}_{e} \mathscr{F}$ as $\left\{R_{x}\right\}$-submodules. Also, for each $F \in \mathscr{F}$, $\left\{R_{(x, \beta)}\right\}$ becomes a system of DA-algebras over $\mathscr{E}_{\beta}$ such that

$$
d \mathcal{O}_{F}: \mathscr{T}_{e} \mathscr{G}_{u n}(q) \rightarrow \mathscr{T}_{e} \mathscr{F}_{u n}(q)
$$

is a homomorphism of $\left\{R_{(x, \beta)}\right\}$-modules.

Secondly, we require that the natural maps

$$
\frac{\mathscr{T}_{e} \mathscr{G}_{u n}(q)}{\mathscr{M}_{\beta} \cdot \mathscr{T}_{e} \mathscr{G}_{u n}(q)} \sim \mathscr{T}_{e} \mathscr{G} \text { and } \quad \frac{\mathscr{T}_{e} \mathscr{F}_{u n}(q)}{\mathscr{M}_{\beta} \cdot \mathscr{T}_{e} \mathscr{F}_{u n}(q)} \sim \mathscr{T}_{e} \mathscr{F}
$$

are isomorphisms of $\left\{R_{x}\right\}$-modules.

Thirdly, we require that

$$
\mathscr{M}_{x} \cdot \mathscr{T}_{e} \mathscr{G} \subset \mathscr{T} \mathscr{G} \text { and } \mathscr{M}_{x} \cdot \mathscr{T}_{e} \mathscr{F} \subset \mathscr{T} \mathscr{F} \text {. }
$$

This last condition implies that both extended tangent spaces $\mathscr{T}_{e} \mathscr{G}$ and $\mathscr{T}_{e} \mathscr{F}$ differ from the tangent spaces $\mathscr{T} \mathscr{G}$ and $\mathscr{T} \mathscr{F}$ by 'constant vector fields'.

Exponential maps. We first define a group $\mathscr{G}_{e q}(q)$ of equivalences of unfoldings with $q$ parameters consisting of those elements of $\mathscr{G}_{u n}(q)$ that are unfoldings of the identity of $\mathscr{G}$ and which involve a diffeomorphism $\Phi$ defined in a neighbourhood of the origin of $\mathbb{R}^{q}$. See $\$ 2 \cdot 2 \cdot 2$ of Damon [3].

Again, we can consider 'unfoldings' of elements of $G_{e q}(q)$ to lie in $G_{e q}(q+1)$ via a one-parameter unfolding of the identity of $G_{e q}(q)$. Computing the extended tangent space at the identity, we have:

$$
\mathscr{T}_{e} \mathscr{G}_{e q}(q)=\mathscr{T}_{e} \mathscr{G}_{u n}(q) \oplus \mathscr{E}_{\beta} \cdot\left\{\frac{\partial}{\partial_{\beta}}\right\} .
$$

The restriction of the exponential map for $\mathscr{K}$ induces a map

where $\partial / \partial t \phi_{t}=\xi . \phi_{t}$ and $\phi_{0}=i d$.

$$
\begin{aligned}
\exp : \mathscr{T}_{e} \mathscr{G}_{e q}(q) & \rightarrow \mathscr{G}_{e q}(q+1) \\
\xi & \mapsto \phi_{t}
\end{aligned}
$$

Filtration preservation. The group $\mathscr{G}_{u n}(q)$ preserves the filtration $\left\{\left\{\mathscr{M}_{x}^{k}\right\} . \mathscr{F}_{u n}(q)\right\}$ on $\mathscr{F}_{u n}(q)$ and it induces an action on the quotient

$$
\frac{\mathscr{F}_{u n}(q)}{\left\{\mathscr{M}_{x}^{k}\right\} \cdot \mathscr{F}_{u n}(q)}, \forall k \geqslant 0
$$


We now show that $\mathscr{G}=\mathscr{K}_{\lambda}^{\Gamma}$ is a geometric subgroup, that is, it satisfies the four conditions listed above. To do so, take $\mathscr{F}=\overrightarrow{\mathscr{E}}_{(x, \lambda)}^{\Gamma}, \mathscr{F}_{u n}(q)=\overrightarrow{\mathscr{E}}_{(x, \lambda, \beta)}$ and

$$
\mathscr{G}_{u n}(q)=\left\{(T, X, \Lambda) \in \mathscr{K}_{\lambda, u n}^{\Gamma}(q) \mid \Phi=\mathbf{I}_{\beta}\right\}
$$

Naturality is clear.

The tangent space condition follows from Section $2 \cdot 2 \cdot 3$ and preliminary results of previous sections.

To verify the exponential map condition we need to define

$$
\mathscr{G}_{e q}(q)=\left\{(T, X, \Lambda) \in \mathscr{K}_{\lambda, u n}^{\Gamma}(q) \mid(T, X, \Lambda) \text { is an unfolding of the identity in } \mathscr{K}_{\lambda}^{\Gamma}\right\} .
$$

Again, we omit the details because everything extends readily to our context.

Finally, the filtration preservation condition holds because it already holds for the non-equivariant situation, and we consider a linear $\Gamma$-action.

The Unfolding and Finite Determinacy theorems are then a rewording in our situation of the corresponding results in the general theory of [3].

\section{Example: $\mathbf{D}_{4}$-equivariant problems}

A $\Gamma$-bifurcation problem is a germ $F \in \overrightarrow{\mathscr{E}}_{(x, \lambda)}^{\Gamma}$ such that $f^{o}=0$ and $f_{x}^{o}=0$. In this section our aim is to solve the recognition problem for $\mathbf{D}_{4}$-bifurcation problems. We identify $\mathbb{R}^{2}$ with $\mathbf{C}$, and in complex notation the action of $\mathbf{D}_{4}$ on $\mathbf{C} \times \mathbf{C}$ is generated by

$$
\begin{gathered}
\hat{\kappa} \cdot(z, \lambda)=(\kappa(z), \lambda)=(\bar{z}, \lambda), \\
\hat{\mu} \cdot(z, \lambda)=(\mu(z), \kappa(\lambda))=(i \bar{z}, \bar{\lambda}),
\end{gathered}
$$

where $\kappa$ denotes the flip $\kappa(z)=\bar{z}$ and $\mu$ denotes the action $\mu(z)=\left(e^{i \frac{\pi}{2}} \kappa\right) \cdot z=i \bar{z}$.

\subsection{Preliminaries}

Define the $\mathbf{D}_{\mathbf{4}}$-invariant functions

$$
\begin{gathered}
N=z \bar{z}, \\
\delta=-\frac{1}{2}\left(z^{2}+\bar{z}^{2}\right), \\
\Delta=\delta^{2}, \\
u_{4}=\lambda_{2}^{2} .
\end{gathered}
$$

Let $\bar{u}=\left(u_{1}, u_{2}, u_{3}, u_{4}, u_{5}\right)$ with $u_{1}=N, u_{2}=\Delta, u_{3}=\lambda_{1}, u_{4}=\lambda_{2}^{2}, \quad u_{5}=\delta \lambda_{2}$ and $\bar{v}=\left(\lambda_{1}, u_{4}\right)$. Note that there is a unique relation

$$
u_{5}^{2}=u_{2} u_{4} \text {. }
$$

Since it is much more convenient to work with free modules we take $u=\left(u_{1}, u_{2}, u_{3}, u_{4}\right)$. From Peters [12] we have the following results.

Proposition $3 \cdot 1 \cdot 1$. 1. $\overrightarrow{\mathscr{E}}_{(z, \lambda)}^{\mathrm{D}_{4}}$ is freely generated by $z, \delta \bar{z}, \lambda_{2} \bar{z}$ and $\delta \lambda_{2} z$ as an $\mathscr{E}_{u^{-}}$ module.

2. $\mathbf{M}_{(z, \lambda)}^{\mathbf{D}_{4}}$ is freely generated as a $\mathscr{E}_{u}$-module by the following linear maps (in complex notation):

$$
\begin{array}{llll}
\hat{S}_{1}(z, \lambda) w=w, & \hat{S}_{3}(z, \lambda) w=\delta \bar{w}, & \hat{S}_{5}(z, \lambda) w=i \omega \bar{w}, & \hat{S}_{7}(z, \lambda) w=-i \delta \omega w \\
\hat{S}_{2}(z, \lambda) w=\lambda_{2} \bar{w}, & \hat{S}_{r}(z, \lambda) w=\delta \lambda_{2} w, & \hat{S}_{6}(z, \lambda) w=-i \lambda_{2} \omega w, & \hat{S}_{8}(z, \lambda) w=i \delta \lambda_{2} \omega \bar{w}
\end{array}
$$


Table 1. Normal forms. Here top-cod denotes the topological $\mathbf{D}_{\mathbf{4}}$-co-dimension and cod the smooth $\mathbf{D}_{4}$-co-dimension

\begin{tabular}{llcc}
\hline Case & \multicolumn{1}{c}{ Normal form } & top-cod & cod \\
\hline $\mathrm{I}_{0}$ & {$\left[m N+\epsilon_{0} \lambda_{1}, \epsilon_{5}, 1,0\right]$} & 0 & 1 \\
$\mathbf{I}_{1}$ & {$\left[m N+\epsilon_{3} N \lambda_{1}+\epsilon_{4} \lambda_{1}^{2}+\alpha, \epsilon_{5}, 1,0\right]$} & 1 & 2 \\
$\mathbf{I}_{2}$ & {$\left[m N+n N u_{4}+\epsilon_{0} \lambda_{1}, \epsilon_{5}, \lambda_{1}+\epsilon_{8} u_{4}+\alpha, 0\right]$} & 1 & 3 \\
II & {$\left[\epsilon_{2} N^{2}+\epsilon_{0} \lambda_{1}+\alpha N, \epsilon_{5}, 1,0\right]$} & 1 & 1 \\
III & {$\left[-\epsilon_{\sigma} N+\epsilon_{0} \lambda_{1}+\alpha N, \epsilon_{5}, 1+\epsilon_{7} N, 0\right]$} & 1 & 1 \\
IV & {$\left[\epsilon_{1} N+\epsilon_{9} N^{2}+\epsilon_{0} \lambda_{1}+\alpha N, \epsilon_{1}+m_{2} \lambda_{1}, 1,0\right]$} & 1 & 2 \\
V & {$\left[\epsilon_{1} N+\epsilon_{0} \lambda_{1}, \epsilon_{6} \Delta+m_{1} \lambda_{1}+\alpha, 1,0\right]$} & 1 & 2 \\
\hline
\end{tabular}

where $\omega=i / \mathbf{4}\left(\bar{z}^{2}-z^{2}\right)$.

3. $\mathscr{E}_{\lambda}^{\mathbf{D}_{4}}=\bar{v}^{*} \mathscr{E}_{v}$ and $\vec{E}_{\lambda}^{\mathbf{D}_{4}}$ is freely generated as an $\mathscr{E}_{\lambda}^{\mathbf{D}_{4}-m o d u l e ~ b y ~}(1,0)$ and $\left(0, \lambda_{2}\right)$.

Thus, $f \in \overrightarrow{\mathscr{E}}_{(z, \lambda)}^{D_{4}}$ may be written as

$$
f(z, \lambda)=p(u) z+q(u) \delta \bar{z}+r(u) \lambda_{2} \bar{z}+s(u) \delta \lambda_{2} z
$$

and we identify $f$ with $[p, q, r, s]$.

Observe also that since $n=m$ we have $\vec{\Theta}_{(z, \lambda)}^{\mathbf{D}_{4,9}}=\overrightarrow{\mathscr{M}}_{(z, \lambda)}^{\mathrm{D}_{4}}$.

\section{3·2. Classification theorems}

THEOREM $3 \cdot 2 \cdot 1$. (Generic normal form).

Let

$$
f=\left[m N+\epsilon_{0} \lambda_{1}, \epsilon_{5}, 1,0\right],
$$

where $\epsilon_{0}= \pm 1, \epsilon_{5}= \pm 1$ and $m \neq \pm 1,0$. Then $f$ has $\mathbf{D}_{4}$-co-dimension 1 (topological codimension $0, m$ is a modal parameter) and all $\mathbf{D}_{4}$-bifurcation problems $h=[p, q, r, s]$ that

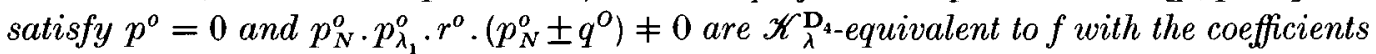
$\epsilon_{0}, \epsilon_{5}$ and $m$ given by $\epsilon_{0}=s g n p_{\lambda_{1}}^{o}, \epsilon_{5}=\operatorname{sgn} q^{o}$ and $m=p_{N}^{o} /\left|q^{o}\right|$.

We now solve the classification problem for the first degeneracies of the generic normal form given in Theorem $3 \cdot 2 \cdot 1$. Let

$$
\Delta_{x, y}(p, q)=p_{x}^{o} q_{y}^{o}-p_{y}^{o} q_{x}^{o}
$$

Theorem 3'2-2. (Classification Theorem). A germ $f=[p, q, r, s] \in \overrightarrow{\mathscr{E}}_{(z, \lambda)}$ is of topological co-dimension 0 or 1 if and only if it belongs to the list shown in Table 1. Moreover, $f$ is

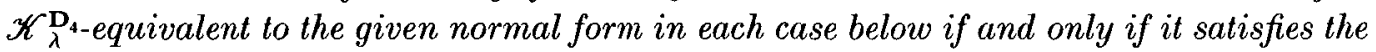
correspondent sets of defining and non-degeneracy conditions listed below in Section $3 \cdot 2 \cdot 1$. In all cases $p^{o}=0$. The parameter $\alpha$ is the unfolding parameter and $m, m_{1}, m_{2}, n$ are moduli.

3:2·1. Additional Information.

Case $I_{1}$. Defining condition : $p_{\lambda_{1}}^{o}=0$.

Non-degeneracy conditions: $p_{N}^{o} \cdot p_{\lambda_{1} \lambda_{1}}^{o} \cdot q^{o} \cdot r^{o} \cdot\left(p_{N}^{o} \pm q^{o}\right)\left(p_{N \lambda_{1}}^{o} q^{o}-q_{\lambda_{1}}^{o} p_{N}^{o}\right) \neq 0$.

Case $I_{2}$. Defining condition: $r^{\circ}=0$.

Non-degeneracy conditions: $q^{o} \cdot p_{\lambda_{1}}^{o} \cdot\left(p_{N}^{o} \pm q^{o}\right) \cdot \Delta_{\lambda_{1}, u_{4}}(p, r) \cdot \xi_{3} \cdot \xi_{4} \neq 0$.

Case II. Defining condition: $p_{N}^{o}=0$.

Non-degeneracy conditions: $p_{N N}^{o} \cdot p_{\lambda_{1}}^{o} \cdot q^{o} \cdot r^{o} \neq 0$. 
Singularity theory

561

Case 1II. Defining condition: $p_{N}^{o}=-q^{o}$.

Non-degeneracy conditions: $p_{\lambda_{1}}^{o} \cdot q^{o} \cdot r^{o} \cdot \xi_{1} \neq 0$.

Case IV. Defining condition: $p_{N}^{o}=q$.

Non-degeneracy conditions: $p_{N}^{o} \cdot p_{\lambda_{1}}^{o} \cdot r^{o} \cdot\left(p_{N N}^{o}+2 p_{\Delta}^{o}-2 q_{N}^{o}\right) \cdot \xi_{2} \neq 0$.

Case V. Defining condition : $q^{0}=0$.

Non-degeneracy conditions: $p_{N}^{o} \cdot p_{\lambda_{1}}^{o} \cdot r^{o} \cdot \Delta_{N, \Delta}(p, q) \cdot \Delta_{N, \lambda_{1}}(p, q) \neq 0$.

Coefficients

$$
\begin{aligned}
& \epsilon_{0}=\operatorname{sgn} p_{\lambda_{1}}^{o} \text {, } \\
& \epsilon_{1}=\operatorname{sgn} p_{N}^{o} \text {, } \\
& \epsilon_{2}=\operatorname{sgn} p_{N N}^{o} \text {, } \\
& \epsilon_{3}=\epsilon_{5} \operatorname{sgn}\left(p_{N \lambda_{1}}^{o} q^{o}-q_{\lambda_{1}}^{o} p_{N}^{o}\right) \text {, } \\
& \epsilon_{4}=\operatorname{sgn} p_{\lambda_{1} \lambda_{1}}^{o} \\
& \epsilon_{5}=\operatorname{sgn} q^{o} \text {, } \\
& \epsilon_{6}=\epsilon_{1} \operatorname{sgn} \Delta_{N, \Delta}(p, q) \text {, } \\
& \epsilon_{7}=\operatorname{sgn} \xi_{1} \text {, } \\
& \epsilon_{8}=\epsilon_{0} \operatorname{sgn} \frac{\left(p_{N}^{o^{2}}-q^{o^{2}}\right) \cdot \Delta_{\lambda_{1}, u_{4}}(p, r)}{p_{\lambda_{1}}^{o} \cdot\left(q^{o} s^{o}-r_{N}^{o} p_{N}^{o}\right)+r_{\lambda_{1}}^{o} \cdot\left(p_{N}^{o^{2}}-q^{o^{2}}\right)}, \\
& \epsilon_{9}=\operatorname{sgn}\left(p_{N N}^{o}+2 p_{\Delta}^{o}-2 q_{N}^{o}\right) \\
& \xi_{1}=-\frac{1}{2 q^{o}}\left(p_{N N}^{o}+2 p_{\Delta}^{o}+2 q_{N}^{o}\right)-\frac{1}{p_{\lambda_{1}}^{o}}\left(p_{N \lambda_{1}}^{o}+q_{\lambda_{1}}^{o}\right)+\frac{1}{r^{o}}\left(s^{o}+r_{N}^{o}\right), \\
& \xi_{2}=p_{\lambda_{1}}^{o} \cdot\left(r_{N}^{o}-s^{o}\right)+r^{o} \cdot\left(q_{\lambda_{1}}^{o}-p_{N \lambda_{1}}^{o}\right) \text {, } \\
& \xi_{3}=p_{N}^{o} \cdot\left(p_{N}^{o^{2}}-q^{o^{2}}\right) \cdot \Delta_{\lambda_{1}, u_{4}}(p, q)+q^{o} \cdot\left(p_{N}^{o^{2}}-q^{o^{2}}\right) \cdot\left(p_{N \lambda_{1}}^{o} p_{u_{4}}^{o}-p_{N u_{4}}^{o} p_{\lambda_{1}}^{o}\right) \\
& \left.+p_{\lambda_{1}}^{o} \cdot q^{o} \cdot p_{N}^{o} \cdot\left(r_{N}^{o^{2}}+s^{o^{2}}\right)-r_{N}^{o} \cdot p_{\lambda_{1}}^{o} \cdot s^{o} \cdot\left(p_{N}^{o^{2}}+q^{o^{2}}\right)\right) \text {, } \\
& \xi_{4}=p_{\lambda_{1}}^{o} \cdot\left(q^{o} s^{o}-p_{N}^{o} r_{N}^{o}\right)+r_{\lambda_{1}}^{o} \cdot\left(p_{N}^{o^{2}}-q^{o^{2}}\right) \text {, } \\
& m=\frac{p_{N}^{o}}{\left|q^{o}\right|} \\
& m_{1}=\frac{\epsilon_{1} \Delta_{N, \lambda_{1}}(p, q)}{\left|p_{\lambda_{1}}^{o}\right| \cdot\left|\Delta_{N, \Delta}(p, q)\right|^{\frac{1}{2}}} \\
& m_{2}=\frac{2\left|q^{o}\right|}{r^{o} \cdot\left|p_{\lambda_{1}}^{o}\right| \cdot\left|p_{N N}^{o}+2 p_{\Delta}^{o}-2 p_{N}^{o}\right|} \xi_{2}, \\
& n=\frac{\epsilon_{5} p_{\lambda_{1}}^{o} \cdot\left(q^{o^{2}}-p_{N}^{o^{2}}\right)}{q^{o^{2}} \cdot\left[p_{\lambda_{1}}^{o} \cdot\left(s^{o} q^{o}-p_{N}^{o} \cdot r_{N}^{o}\right)+r_{\lambda_{1}}^{o} \cdot\left(p_{N}^{o}-q^{o^{2}}\right)\right]^{2}} \xi_{3} .
\end{aligned}
$$

3.3. Proofs

3:3.1. Tangent spaces. The $\mathbf{D}_{4}$-extended tangent space at $f \in \overrightarrow{\mathscr{E}}_{(z, \lambda)}$, defined in Section 2 , is given by

$$
\mathscr{T}_{e}^{\mathbf{D}_{4}}(f)=\mathscr{E}_{u} \cdot\left\langle g_{1} \ldots g_{12}\right\rangle+\mathscr{E}_{\left(\lambda_{1}, u_{4}\right)} \cdot\left\langle g_{13}, g_{14}\right\rangle
$$


562 Jacques-Élie Furter, Angela Maria Sitta and Ian Stewart where

$$
\begin{aligned}
& g_{1}=[p, q, r, s] \text {, } \\
& g_{2}=\left[u_{4} r, u_{4} s, p, q\right] \text {, } \\
& g_{3}=[\Delta q, p, \Delta s, r] \text {, } \\
& g_{4}=[N p-\Delta q, p-N q,-N r+\Delta s,-r+N s], \\
& g_{5}=[\Delta p-N \Delta q, N p-\Delta q, N \Delta s-\Delta r, \Delta s-N r] \text {, } \\
& g_{6}=\left[-N u_{4} r+\Delta u_{4} s,-u_{4} r+N u_{4} s, N p-\Delta q, p-N q\right] \text {, } \\
& g_{7}=\left[\Delta u_{4} s, u_{4} r, \Delta q, p\right] \text {, } \\
& g_{8}=\left[-\Delta u_{4} r+N \Delta u_{4} s,-N u_{4} r+\Delta u_{4} s, \Delta p-N \Delta q, N p-\Delta q\right] \text {, } \\
& g_{9}=\left[2 N p_{N}+4 \Delta p_{\Delta}+p, 2 N q_{N}+4 \Delta q_{\Delta}+3 q\right. \text {, } \\
& \left.2 N r_{N}+4 \Delta r_{\Delta}+r, 2 N s_{N}+4 \Delta s_{\Delta}+3 s\right] \text {, } \\
& g_{10}=\left[-2 \Delta p_{N}-4 N \Delta p_{\Delta}+\Delta q,-2 \Delta q_{N}-4 N \Delta q_{\Delta}-2 N q+p\right. \text {, } \\
& \left.-2 \Delta r_{N}-4 N \Delta r_{\Delta}+\Delta s,-2 \Delta s_{N}-4 N \Delta s_{\Delta}-2 N s+r\right] \text {, } \\
& g_{11}=\left[-2 \Delta u_{4} s_{N}-4 N \Delta u_{4} s_{\Delta}-2 N u_{4} s+u_{4} r,-2 u_{4} r_{N}-4 N u_{4} r_{\Delta}+u_{4} s\right. \text {, } \\
& \left.-2 \Delta q_{N}-4 N \Delta q_{\Delta}-2 N q+p,-2 p_{N}-4 N p_{\Delta}+q\right] \text {, } \\
& g_{12}=\left[2 N \Delta u_{4} s_{N}+4 \Delta^{2} u_{4} s_{\Delta}+3 \Delta u_{4} s, 2 N u_{4} r_{N}+4 \Delta u_{4} r_{\Delta}+u_{4} r\right. \text {, } \\
& \left.2 N \Delta q_{N}+4 \Delta^{2} q_{\Delta}+3 \Delta q, 2 N p_{N}+4 \Delta p_{\Delta}+p\right] \text {, } \\
& g_{13}=\left[p_{\lambda_{1}}, q_{\lambda_{1}}, r_{\lambda_{1}}, s_{\lambda_{1}}\right] \text {, } \\
& g_{14}=\left[2 u_{4} p_{u_{4}}, 2 u_{4} q_{u_{4}}, r+2 u_{4} r_{u_{4}}, s+2 u_{4} s_{u_{4}}\right] \text {. }
\end{aligned}
$$

Similarly, with $\left\{g_{i}\right\}_{i-1}^{14}$ as previously defined,

$$
\begin{gathered}
\mathscr{T} \mathscr{U}^{\mathbf{D}_{4}}(f)=\mathscr{E}_{u} \cdot\left\langle N g_{1}, \Delta g_{1}, \lambda_{1} g_{1}, u_{4} g_{1}, g_{2}, g_{3}, g_{4}, g_{5}, g_{6}, g_{7}, g_{8},\right. \\
\left.N g_{9}, \Delta g_{9}, \lambda_{1} g_{9}, u_{4} g_{9}, g_{10}, g_{11}, g_{12}\right\rangle+\mathscr{E}_{\left(\lambda_{1}, u_{4}\right)} \cdot\left\langle\lambda_{1}^{2} g_{13}, u_{4} g_{13}, \lambda_{1} g_{14}, u_{4} g_{14}\right\rangle, \\
\mathscr{T}{ }_{e}^{\mathbf{D}_{4}}(f)=\mathscr{T} \mathscr{U}^{\mathbf{D}_{4}}(f)+\mathbb{R} .\left\langle g_{1}, g_{9}, g_{13}, \lambda_{1} g_{13}, g_{14}\right\rangle, \\
\mathscr{R} \mathscr{T} \mathscr{U}^{\mathbf{D}_{4}}(f)=\mathscr{E}_{u} \cdot\left\langle N g_{1}, \Delta g_{1}, \lambda_{1} g_{1}, u_{4} g_{1}, g_{2}, g_{3}, g_{4}, g_{5}, g_{6}, g_{7}, g_{8},\right. \\
\left.N g_{9}, \Delta g_{9}, \lambda_{1} g_{9}, u_{4} g_{9}, g_{10}, g_{11}, g_{12}\right\rangle .
\end{gathered}
$$

We define the $\mathbf{D}_{4}$-topological codimension of a $\mathbf{D}_{4}$-bifurcation problem as its $\mathbf{D}_{4}$-codimension minus the number of modal parameters. A modal parameter changes the differentiable type of the singularity but not its topological type, see Golubitsky and Schaeffer [8].

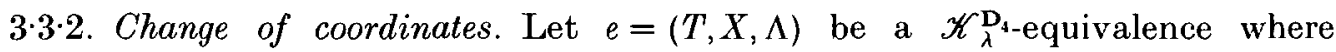
$X=[a, b, c, d]$ with $a, b, c, d \in \mathscr{E}_{u}$ and $a^{o}>0, \Lambda=\left(\Lambda_{1}, \lambda_{2} \Lambda_{2}\right)$ with $\Lambda_{1} \in \mathscr{M}_{\lambda}^{\mathbf{D}_{4}},\left(\Lambda_{1}\right)_{\lambda_{\mathrm{t}}}^{o}>0$ and $\Lambda_{2} \in \mathscr{E}_{\lambda}^{\mathbf{D}_{4}}$ and $T=\sum_{i=1}^{8} A_{i} \hat{S}_{i}$ with $A_{i} \in \mathscr{E}_{u}$ and $A_{1}^{o}>0$.

To simplify the notation, we define the following expressions:

$$
\begin{aligned}
& \tilde{N}_{1}=a^{2} N-2 a b \Delta+b^{2} \Delta N+c^{2} N u_{4}-2 c d \Delta u_{4}+d^{2} N \Delta u_{4} \\
& \tilde{N}_{2}=-2 a c+2 a d N+2 b c N-2 b d \Delta \\
& D_{1}=a^{2}-2 a b N+b^{2} \Delta+c^{2} u_{4}-2 c d N u_{4}+d^{2} \Delta u_{4} \\
& D_{2}=-2 a c N+2 a d \Delta+2 b c \Delta-2 b d N \Delta \\
& \tilde{\Delta}_{1}=D_{1}^{2} \Delta+D_{2}^{2} u_{4} \quad \text { and } \quad \tilde{\Delta}_{2}=2 D_{1} D_{2}
\end{aligned}
$$


Using (3.3) below we find that

$$
\left.\begin{array}{rl}
\tilde{N} & =N \circ X=\tilde{N}_{1}+\delta \lambda_{2} \tilde{N}_{2} \\
\tilde{\Delta} & =\Delta \circ X=\tilde{\Delta}_{1}+\delta \lambda_{2} \tilde{\Delta}_{2} \\
\tilde{\lambda}_{1} & =\lambda_{1} \circ \Lambda=\Lambda_{1} \\
\tilde{u}_{4} & =u_{4} \circ \Lambda=u_{4} \Lambda_{2}^{2} \\
\tilde{\delta} & =\delta \circ X=D_{1} \delta+D_{2} \lambda_{2} .
\end{array}\right\}
$$

For $h \in \mathscr{E}_{u}$, we define $\tilde{h}=h\left(\tilde{N}, \tilde{\Delta}, \tilde{\lambda}_{1}, \tilde{u}_{4}\right)$.

Let $f \in \overrightarrow{\mathscr{E}}_{(z, \lambda)}$ given by $f=[p, q, r, s]$. We consider the change of coordinates in two steps : first $f^{\prime}=\left(\mathbf{I}_{2}, X, \Lambda\right) \cdot f$, and then $f^{\prime \prime}=\left(T, \mathbf{I}_{2}, \mathbf{I}_{2}\right) \cdot f^{\prime}$, to produce the final result. We refer to [14] for a complete set of data. Here we give only the information needed to compute the intrinsic submodules. A calculation shows that

(i) $f^{\prime}=\left(\mathbf{I}_{2}, X, \Lambda\right) \cdot f=\left[p^{\prime}, q^{\prime}, r^{\prime}, s^{\prime}\right]$, where

$$
\left.\begin{array}{l}
p^{\prime} \in\langle\tilde{p}\rangle+\langle\Delta \tilde{q}\rangle+\mathscr{M}_{N, \Delta)} \cdot\left\langle u_{4} \tilde{q}\right\rangle+\left\langle u_{\mathbf{4}} \tilde{r}\right\rangle+\mathscr{M}_{(N, \Delta)} \cdot\left\langle u_{\mathbf{4}} \tilde{s}\right\rangle, \\
q^{\prime} \in\langle\tilde{p}\rangle+\langle\tilde{q}\rangle+\left\langle u_{r} \tilde{r}\right\rangle+\left\langle u_{4} \tilde{s}\right\rangle, \\
r^{\prime} \in\langle\tilde{p}\rangle+\mathscr{M}_{(N, \Delta)} \cdot\langle\tilde{q}\rangle+\langle\tilde{r}\rangle+\langle\Delta \tilde{s}\rangle+\mathscr{M}_{N, \Delta)} \cdot\left\langle u_{4} \tilde{s}\right\rangle, \\
s^{\prime} \in\langle\tilde{p}\rangle+\langle\tilde{q}\rangle+\langle\tilde{r}\rangle+\langle\tilde{s}\rangle .
\end{array}\right\}
$$

(ii) $f^{\prime \prime}=\left(T, \mathbf{I}_{2}, \mathbf{I}_{2}\right) \cdot f^{\prime}=\left[p^{\prime \prime}, q^{\prime \prime}, r^{\prime \prime}, s^{\prime \prime}\right]$, where

$$
\begin{aligned}
p^{\prime \prime}= & \left(A_{1}+A_{4} N+A_{5} \Delta\right) p^{\prime}+\left(A_{3}-A_{4}-A_{5} N\right) \Delta q^{\prime}+\left(A_{2}-A_{6} N-A_{8} \Delta\right) u_{4} r^{\prime} \\
& +\left(A_{6}+A_{7}+A_{8} N\right) \Delta u_{4} s^{\prime}, \\
q^{\prime \prime}= & \left(A_{3}+A_{4}+A_{5} N\right) p^{\prime}+\left(A_{1}-A_{4} N-A_{5} \Delta\right) q^{\prime}+\left(A_{7}-A_{6}-A_{8} N\right) u_{4} r^{\prime} \\
& +\left(A_{2}+A_{6} N+A_{8} \Delta\right) u_{4} s^{\prime} \\
r^{\prime \prime}= & \left(A_{2}+A_{6} N+A_{8} \Delta\right) p^{\prime}+\left(A_{7}-A_{6}-A_{8} N\right) \Delta q^{\prime}+\left(A_{1}-A_{4} N-A_{5} \Delta\right) r^{\prime} \\
& +\left(A_{3}+A_{4}+A_{5} N\right) \Delta s^{\prime}, \\
s^{\prime \prime}= & \left(A_{6}+A_{7}+A_{8} N\right) p^{\prime}+\left(A_{2}-A_{6} N-A_{8} \Delta\right) q^{\prime}+\left(A_{3}-A_{4}-A_{5} N\right) r^{\prime} \\
& +\left(A_{1}+A_{4} N+A_{5} \Delta\right) s^{\prime} .
\end{aligned}
$$

$3 \cdot 3 \cdot 3$. Intrinsic ideals and submodules. Recall that an intrinsic submodule of $\overrightarrow{\mathscr{E}}_{(z, \lambda)}$ is a submodule that is invariant under the action of $\mathscr{K}_{\lambda}^{\mathbf{D}_{4}}$ and an intrinsic ideal in $\overrightarrow{\mathscr{E}} \mathrm{D}_{(z, \lambda)}$ is invariant under the group of coordinate changes $(x, \lambda) \mapsto(X(x, \lambda), \Lambda(\lambda))$ where $X$ and $\Lambda$ satisfy the conditions of Section $3 \cdot 3 \cdot 2$. Clearly, $\left(\dot{M}_{(z, \lambda)}^{\mathrm{D}_{4}}\right)^{k}$ is intrinsic for all $k$. Moreover, it follows from (3.2) that sums and products of the intrinsic ideals $\left\langle\lambda_{1}, u_{4}\right\rangle$ and $\left\langle u_{4}\right\rangle$ are also intrinsic.

Note that intrinsic modules must be separately intrinsic for the actions of $\left(T, \mathbf{I}_{2}, \mathbf{I}_{2}\right)$ and $\left(\mathbf{I}_{2}, X, \lambda\right)$. For the first case, we can give a set of necessary and sufficient criteria (Lemma $3 \cdot 3 \cdot 1$ ). We cannot be so precise for the latter, but we can derive formulas that will be useful in the explicit calculations. We define a subgroup of $\mathscr{K}_{\lambda}^{D_{4}}$ by

$$
\mathscr{C}_{\lambda}^{\mathbf{D}_{4}}=\left\{\left(T, \mathbf{I}_{2}, \mathbf{I}_{2}\right) \mid\left(T, \mathbf{I}_{2}, \mathbf{I}_{2}\right) \in \mathscr{K}_{\lambda}^{\mathbf{D}_{4}}\right\}
$$

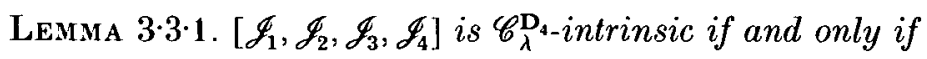

$$
\begin{array}{lll}
\left\langle\Delta u_{4}\right\rangle \mathscr{I}_{4} \subset\langle\Delta\rangle \mathscr{I}_{2} \subset \mathscr{I}_{1} \subset \mathscr{I}_{2} \subset \mathscr{I}_{4}, & \langle\Delta\rangle \mathscr{I}_{4} \subset \mathscr{I}_{3}, & \langle\Delta\rangle \mathscr{I}_{2} \subset \mathscr{I}_{3}, \\
\left\langle\Delta u_{4}\right\rangle \mathscr{I}_{4} \subset\left\langle u_{4}\right\rangle \mathscr{I}_{3} \subset \mathscr{I}_{1} \subset \mathscr{I}_{3} \subset \mathscr{I}_{4}, & \left\langle u_{4}\right\rangle \mathscr{I}_{4} \subset \mathscr{I}_{2}, & \left\langle u_{4}\right\rangle \mathscr{I}_{3} \subset \mathscr{I}_{2} .
\end{array}
$$


564 Jacques-Élie Furter, Angela Maria Sitta and Ian Stewart

Proof. This follows by straightforward calculations using (3-4).

Now, define the following ideals

$$
\begin{aligned}
& \mathscr{I}_{2 k}=\mathscr{M}_{(N, \Delta)}^{2 k}+\mathscr{M}_{(N, \Delta)}^{2 k-2} \Delta u_{4}+\ldots \Delta^{k} u_{4}^{k}, \\
& \mathscr{E}_{k}=\Delta^{k}+\Delta^{k-1} \mathscr{M}_{(N, \Delta)}^{2} u_{4}+\ldots+\mathscr{M}_{(N, \Delta)}^{2 k} u_{4}^{k} \text {. }
\end{aligned}
$$

We verify by induction on $k \geqslant 1$ that

$$
\begin{aligned}
& \tilde{\mathscr{M}}_{(N, \Delta)}^{2 k} \subset \mathscr{I}_{2 k}+\left(\mathscr{M}_{(N, \Delta)} \cdot \mathscr{J}_{2 k-2}\right) \delta \lambda_{2}, \\
& \langle\tilde{\Delta}\rangle^{k} \subset \mathscr{I}_{k}+\left(\mathscr{M}_{(N, \Delta)} \cdot \mathscr{I}_{k-1}\right) \delta \lambda_{2}, \\
& \tilde{M}_{(N, \Delta)}^{2 k+1} \subset \mathscr{M}_{(N, \Delta)} \cdot \mathscr{I}_{2 k}+\mathscr{I}_{2 k} \delta \lambda_{2} .
\end{aligned}
$$

3.3.4. Proof of Theorem $3 \cdot 2 \cdot 1$. The proof of Theorem $3 \cdot 2 \cdot 1$ is a consequence of the following lemma.

Lemma 3:3.2. Let $f=\left[m N+\epsilon_{0} \lambda_{1}, \epsilon_{5}, 1,0\right]$ as defined above. Then the $\mathbf{D}_{\mathbf{4}}$-unipotent tangent space is intrinsic and is given by

$$
\mathscr{T} \mathscr{U}^{\mathbf{D}_{4}}(f)=\left[\mathscr{M}_{u}^{2}+\left\langle\Delta, u_{4}\right\rangle, \mathscr{M}_{u}, \mathscr{M}_{u}, \mathscr{E}_{u}\right] .
$$

Proof. A calculation shows that, since $m \neq \pm 1,0$,

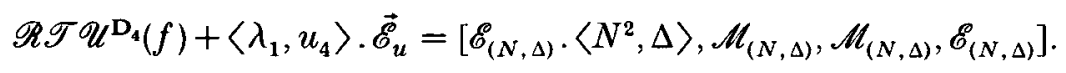

It follows that

$$
\frac{\overrightarrow{\mathscr{E}}_{u}}{\mathscr{R} \mathscr{T} \mathscr{U}^{\mathbf{D}_{4}}(f)+\left\langle\lambda_{1}, u_{4}\right\rangle \cdot \overrightarrow{\mathscr{E}}_{u}}
$$

is generated by $[N, 0,0,0],[1,0,0,0],[0,1,0,0]$ and $[0,0,1,0]$ as a real vector space.

Let $R=\left[\mathscr{M}_{u}^{2}+\left\langle\Delta, u_{4}\right\rangle, \mathscr{M}_{u}, \mathscr{M}_{u}, \mathscr{E}_{u}\right]$. We will show that $R=\mathscr{T} \mathscr{U}^{\mathbf{D}_{4}}(f)$.

(i) $\mathscr{T} \mathscr{U}^{\mathbf{D}_{4}}(f) \subset R$

From the Preparation Theorem,

$$
\frac{\vec{E}_{u}}{\mathscr{R} \mathscr{T}^{\mathbf{D}_{4}}(f)}
$$

is generated by $[N, 0,0,0],[1,0,0,0],[0,1,0,0]$ and $[0,0,1,0]$ as an $\mathscr{E}_{\left(\lambda_{1}, u_{4}\right)}$-module. Therefore, $\phi \in R$ can be represented as

$$
\phi=\phi_{1}[N, 0,0,0]+\phi_{2}[1,0,0,0]+\phi_{3}[0,1,0,0]+\phi_{4}[0,0,1,0]+\tilde{\phi}
$$

where $\left\{\phi_{i}\right\}_{i=1}^{4} \subset \mathscr{E}_{\left(\lambda_{1}, u_{4}\right)}$ and $\tilde{\phi} \in \mathscr{R} \mathscr{T} \mathscr{U}^{\mathbf{D}_{4}}(f)$. Since $\mathscr{R} \mathscr{T} \mathscr{U}^{\mathbf{D}_{4}}(f) \in R$, this implies that $\left[\phi_{1} N+\phi_{2}, \phi_{3}, \phi_{4}, 0\right] \in R$ and so $\phi_{1}, \phi_{3}, \phi_{4} \in \mathscr{M}_{\left(\lambda_{1}, u_{4}\right)}$ and $\phi_{2} \in \mathscr{E}_{\left(\lambda_{1}, u_{4}\right)} \cdot\left\langle\lambda_{1}^{2}, u_{4}\right\rangle$. Hence, as an $\mathscr{E}_{\left(\lambda_{1}, u_{4}\right)}$-module,

$$
\frac{R}{\mathscr{R} \mathscr{T} \mathscr{U}^{\mathbf{D}_{4}}(f)}
$$

is generated by

$$
\left.\begin{array}{llll}
{\left[N \lambda_{1}, 0,0,0\right],} & {\left[N u_{4}, 0,0,0\right],} & {\left[\lambda_{1}^{2}, 0,0,0\right],} & {\left[u_{4}, 0,0,0\right],} \\
{\left[0, \lambda_{1}, 0,0\right],} & {\left[0, u_{4}, 0,0\right],} & {\left[0,0, \lambda_{1}, 0\right],} & {\left[0,0, u_{4}, 0\right] .}
\end{array}\right\}
$$


Thus

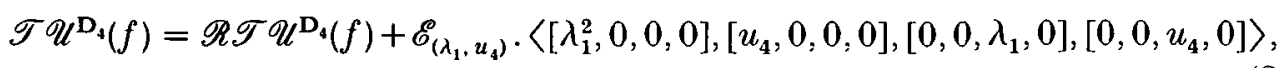

showing that $\mathscr{T} \mathscr{U}^{\mathbf{D}_{4}}(f) \subset R$.

(ii) $R \subset \mathscr{T} \mathscr{U}^{\mathbf{D}_{4}}(f)$

Step 1. Consider the following set of generators obtained from $\mathscr{R} \mathscr{T} \mathscr{U}^{\mathbf{D}_{4}}(f)$ modulo $\mathscr{E}_{\left(\lambda_{1}, u_{4}\right)} \cdot\left\langle\left[\lambda_{1}^{2}, 0,0,0\right],\left[u_{4}, 0,0,0\right],\left[0,0, \lambda_{1}, 0\right],\left[0,0, u_{4}, 0\right]\right\rangle$, provided that $m \neq \pm 1,0$.

$$
\begin{aligned}
h_{1}^{\prime} & =\left[m N^{2}+\epsilon_{0} N \lambda_{1}, \epsilon_{5} N, N, 0\right], \\
h_{2}^{\prime} & =\left[m N \Delta+\epsilon_{0} \Delta \lambda_{1}, \epsilon_{5} \Delta, \Delta, 0\right], \\
h_{3}^{\prime} & =\left[m N \lambda_{1}, \epsilon_{5} \lambda_{1}, 0,0\right], \\
h_{4}^{\prime} & =\left[m N u_{4}, \epsilon_{5} u_{4}, 0,0\right], \\
h_{5}^{\prime} & =\left[0,0, m N, \epsilon_{5}\right], \\
h_{6}^{\prime} & =\left[\epsilon_{5} \Delta, m N+\epsilon_{0} \lambda_{1}, 0,1\right], \\
h_{7}^{\prime} & =\left[0,\left(m-\epsilon_{5}\right) N+\epsilon_{0} \lambda_{1},-N, 0\right], \\
h_{8}^{\prime} & =\left[\left(m-\epsilon_{5}\right) N \Delta+\epsilon_{0} \Delta \lambda_{1}, m N^{2}+\epsilon_{0} N \lambda_{1}-\epsilon_{5} \Delta,-\Delta,-N\right], \\
h_{9}^{\prime} & =\left[-N u_{4},-u_{4}, m N^{2}+\epsilon_{0} N \lambda_{1}-\epsilon_{5} \Delta,\left(m-\epsilon_{5}\right) N+\epsilon_{0} \lambda_{1}\right], \\
h_{10}^{\prime} & =\left[0, u_{4}, \epsilon_{5} \Delta, m N+\epsilon_{0} \lambda_{1}\right], \\
h_{11}^{\prime} & =\left[-\Delta u_{4},-N u_{4},\left(m-\epsilon_{5}\right) N \Delta+\epsilon_{0} \Delta \lambda_{1}, m N^{2}+\epsilon_{0} N \lambda_{1}-\epsilon_{5} \Delta\right], \\
h_{12}^{\prime} & =\left[\epsilon_{0} N \lambda_{1}, 0, N, 0\right], \\
h_{13}^{\prime} & =\left[\epsilon_{0} \Delta \lambda_{1}, 0, \Delta, 0\right], \\
h_{14}^{\prime} & =\left[m \Delta, \epsilon_{5} N, 0,0\right], \\
h_{15}^{\prime} & =\left[0,0, \epsilon_{5} N, m\right], \\
h_{16}^{\prime} & =\left[0,0, \epsilon_{5} \Delta, m N\right] . \\
&
\end{aligned}
$$

Step 2 . Let $I$ be the $\mathscr{E}_{\left(\lambda_{1}, u_{4}\right)}$-module generated by $\left\{h_{1}^{\prime} \ldots h_{16}^{\prime}, \Delta h_{12}^{\prime}, \Delta h_{15}^{\prime}, N h_{6}^{\prime}, N h_{14}^{\prime}\right\}$ and let $S$ be the $\mathscr{E}_{\left(\lambda_{1}, u_{4}\right)}$-module generated by

$$
\begin{aligned}
& \left\{\left[N^{2}, 0,0,0\right],\left[N \lambda_{1}, 0,0,0\right],[N \Delta, 0,0,0],[\Delta, 0,0,0],\left[N u_{4}, 0,0,0\right],[0, N, 0,0],\right. \\
& {[0, \Delta, 0,0],\left[0, \lambda_{1}, 0,0\right],\left[0, N^{2}, 0,0\right],\left[0, u_{4}, 0,0\right],[0,0, N, 0],\left[0,0, N^{2}, 0\right],[0,0, \Delta, 0],} \\
& \left.[0,0, N \Delta, 0],[0,0,0,1],[0,0,0, N],\left[0,0,0, N^{2}\right],[0,0,0, \Delta]\right\} .
\end{aligned}
$$

It is straightforward to show that $I \subset S$, and a calculation shows that $I+\mathscr{M}_{\left(\lambda_{1}, u_{4}\right)} \cdot S=S$. By Nakayama's Lemma, $S \subset I$ and so $I=S$. From (3.5) and (3.6),

$$
\frac{R}{\mathscr{T} \mathscr{U}^{\mathbf{D}_{4}}(f)}=\mathscr{E}_{\left(\lambda_{1}, u_{4}\right)} \cdot\left\langle\left[N \lambda_{1}, 0,0,0\right],\left[N u_{4}, 0,0,0\right],\left[0, \lambda_{1}, 0,0\right],\left[0, u_{4}, 0,0\right]\right\rangle .
$$

Therefore,

$$
\frac{R}{\mathscr{T} \mathscr{U}^{\mathbf{D}_{4}}(f)} \subset I \subset \mathscr{T} \mathscr{U}^{\mathbf{D}_{4}}(f),
$$

that is $R \subset \mathscr{T} \mathscr{U}^{\mathrm{D}_{4}}(f)$ and, from (i) and (ii),

$$
\mathscr{T} \mathscr{U}^{\mathrm{D}}(f)=R=\left[\mathscr{M}_{u}^{2}+\left\langle\Delta, u_{4}\right\rangle, \mathscr{M}_{u}, \mathscr{M}_{u}, \mathscr{E}_{u}\right] .
$$


566 Jacques-Ét.te Furter, Angela Maria Sitta and Ian Stewart

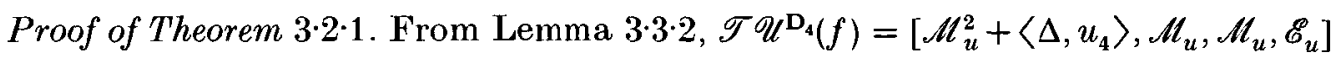
and $\mathscr{T}_{e}^{\mathrm{D}_{4}}(f)=\mathscr{T} \mathscr{U}^{\mathrm{D}_{4}}(f)+\mathbb{R} .\left\{g_{1}, g_{9}, g_{13}, \lambda_{1} g_{13}, g_{14}\right\}$. Hence

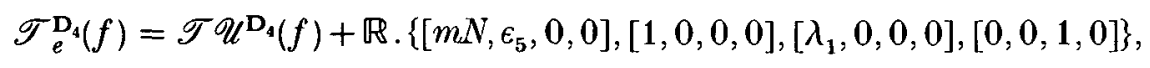

so that $\overrightarrow{\mathscr{E}}_{(2, \lambda)}^{\mathrm{D}_{4}}=\mathscr{T}_{e}^{\mathbf{D}_{4}}(f)+\mathbb{R} .\{[N, 0,0,0]\}$. Therefore, $f$ has $\mathbf{D}_{4}$-co-dimension 1 and topological codimension 0 since $m$ is a modal parameter.

From Lemma 3.3.1 and Proposition 2.5.2, $\mathscr{T} \mathscr{U}^{\mathbf{D}_{4}}(f)=\operatorname{Intr} \mathscr{T} \mathscr{U}^{\mathbf{D}_{4}}(f) \subset \mathbf{P}(f)$.

Following the classical framework, we make explicit changes of coordinates modulo $\mathscr{T} \mathscr{U}^{\mathbf{D}_{4}}(f)$, which is contained in the set of higher order terms of $f$. For $h=[p, q, r, s] \in \mathscr{E}_{(z, \lambda)}^{D_{4}}$, let $\hat{h}=h$ modulo terms in $\mathscr{T} \mathscr{U}^{\mathrm{D}_{4}}(f)$. Then,

$$
\hat{h}=\left[p_{N}^{o} N+p_{\lambda_{1}}^{o} \lambda_{1}, q^{o}, r^{o}, 0\right]
$$

and it is a routine calculation to show that $\hat{h}$ can be reduced into the normal form $f$ $=\left[m N+\epsilon_{0} \lambda_{1}, \epsilon_{5}, 1,0\right]$ if $p^{o}=0$ and $p_{N}^{o} \cdot p_{\lambda_{1}}^{o} \cdot r^{o} \cdot\left(p_{N}^{o} \pm q^{o}\right) \neq 0$, with

$$
m=\frac{p_{N}^{o}}{\left|q^{o}\right|}, \quad \epsilon_{0}=\operatorname{sgn} p_{\lambda_{1}}^{o} \quad \text { and } \quad \epsilon_{5}=\operatorname{sgn} q^{o} .
$$

335. Pre-normal form. Before proving the Classification Theorem, we present some preliminary results to obtain a pre-normal form for $\mathbf{D}_{4}$-bifurcation problems $f=[p, q, r, s]$ when $q^{o} \neq 0$. By this we mean a simplified form for the germ, which goes part way towards the final normal form that will be derived and paves the way for obtaining it. These results are very useful for the calculation of higher codimensions cases and can be readily generalized to other groups.

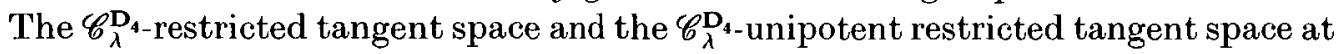
$f$ denoted, respectively by $\mathscr{R}_{e}^{\mathscr{\ell}}(f)$ and $\mathscr{R} \mathscr{T} \mathscr{U}^{\mathscr{E}}(f)$ are

and

$$
\mathscr{R} \mathscr{T}_{e}^{\mathscr{C}}(f)=\left\{T f \mid T \in \mathbf{M}_{(z, \lambda)}^{\mathrm{D}_{4}}\right\}=\left\langle f, T_{2} f \ldots T_{8} f\right\rangle
$$

$$
\mathscr{R} \mathscr{T} \mathscr{U}^{\mathscr{Q}}(f)=\left\{T f \mid T \in \mathbf{M}_{(z, \lambda)}^{\mathrm{D}_{4}} \text { with } T^{o}=0\right\}=\overrightarrow{\mathscr{M}}_{(z, \lambda)}^{\mathrm{D}_{4}} \cdot\langle f\rangle+\left\langle T_{2} f \ldots T_{8} f\right\rangle,
$$

where $T_{1}=\mathbf{I}_{2}, T_{2} \ldots T_{8}$ are the associated real matrices to the linear maps $\hat{S}_{i}, 1 \leqslant i \leqslant$ 8, defined in Proposition 3·1. Specifically,

$$
\begin{aligned}
\mathscr{R} \mathscr{T}_{e}^{\mathscr{C}}(f) & =\left\langle g_{1} \cdot g_{2} \ldots g_{8}\right\rangle, \\
\mathscr{R} \mathscr{T} \mathscr{U}^{\mathscr{E}}(f) & =\left\langle N g_{1}, \Delta g_{1}, \lambda_{1} g_{1}, u_{4} g_{1}, g_{2} \ldots g_{8}\right\rangle,
\end{aligned}
$$

where the $\left\{g_{i}\right\}_{i=1}^{8}$ are previously defined.

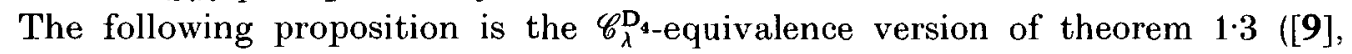
p. 168).

Proposition 3.3.3. Let $f \in \overrightarrow{\mathscr{E}}_{(z, \lambda)}$ and $p$ any germ in $\overrightarrow{\mathscr{E}}_{(2, \lambda)}$. Suppose that

$$
\mathscr{R} \mathscr{T}_{e}^{\mathscr{Q}}(f+t p)=\mathscr{R} \mathscr{T}_{e}^{\mathscr{C}}(f)
$$

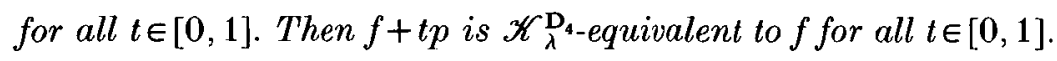

Proof. The proof is similar to the proof of theorem 1.3 mentioned above and it is done in detail in $\left([9], \S 2^{*}\right.$, p. 172). The basic idea is to prove that the argument in Proposition $3 \cdot 3 \cdot 3$ is true in a neighbourhood of $t=\bar{t}$, for all $\bar{t} \in[0,1]$. The result follows from compactness and connectedness of the interval. 
Proposition 3.3.4. Let f be a $\mathbf{D}_{4}$-bifurcation problem and $p \in \overrightarrow{\mathscr{E}}_{(z, \lambda)}$. If $p \in \mathscr{R} \mathscr{T} \mathscr{U}^{\mathscr{E}}(f)$ then $\mathscr{R} \mathscr{T}_{e}^{\mathscr{L}}(f+t p)=\mathscr{R} \mathscr{T}_{e}^{\mathscr{C}}(f), \forall t \in[0,1]$.

Proof. If $p \in \mathscr{R T} \mathscr{T} \mathscr{U}^{\mathscr{B}}(f)$ then

$$
p(z, \lambda)=\sum_{i=1}^{4} \alpha_{i}(u) u_{i} f+\sum_{j=2}^{8} \beta_{j}(u) T_{j} f
$$

with $\alpha_{i}, \beta_{j} \in \mathscr{E}_{u}$. The generators of $\mathscr{R} \mathscr{T}_{e}^{\mathscr{Q}}(f+t p)$ are sums of generators from $\mathscr{R} \mathscr{T}_{e}^{\mathscr{C}}(f)$ and generators of $t . \mathscr{R} \mathscr{T}_{e}^{\mathscr{Q}}(p)$. The inclusions

$$
\mathscr{R} \mathscr{T}_{e}^{\mathscr{Q}}(p) \subset \mathscr{R} \mathscr{T}_{e}^{\mathscr{Q}}(f)+\overrightarrow{\mathscr{M}}_{(z, \lambda)}^{\mathrm{D}_{4}} \cdot \mathscr{R}_{e}^{\mathscr{C}}(f)
$$

and

$$
\mathscr{R} \mathscr{T}_{e}^{\mathscr{Q}}(f+p) \subset \mathscr{R}_{e}^{\mathscr{\mathscr { C }}}(f)
$$

are straightforward. By Nakayama's Lemma $\mathscr{R T}_{e}^{\mathscr{\ell}}(f) \subset \mathscr{R}_{e}^{\mathscr{C}}(f+t p)$, since

$$
\mathscr{R T}_{e}^{\mathscr{C}} \subset \mathscr{R T}_{e}^{\mathscr{E}}(f+t p)+\overrightarrow{\mathscr{M}}_{(z, \lambda)}^{\mathrm{D}_{4}} \cdot \mathscr{R}_{e}^{\mathscr{G}}(f) \text {. }
$$

Therefore, $\mathscr{R T}_{e}^{\mathscr{E}}(f+t p)=\mathscr{R}_{e}^{\mathscr{\ell}}(f), \forall t \in[0,1]$.

CoRollaRY 3.3.5. Let $f$ be a $\mathbf{D}_{4}$-bifurcation problem and $p$ any germ in $\overrightarrow{\mathscr{E}}_{(2, \lambda)}$. If

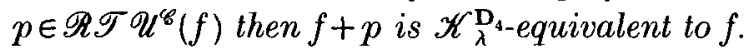

Proof. This follows from Propositions $3 \cdot 3 \cdot 3$ and $3 \cdot 3 \cdot 4$ by setting $t=1$.

As a consequence we have the following result about pre-normal forms.

Theorem 3·3 6. Let $f=[p, q, r, s]$ be a $\mathbf{D}_{4}$-bifurcation problem. If $q^{o} \neq 0$ then fis $\mathscr{K}_{\lambda}^{\mathbf{D}_{4-}}$ equivalent to a pre-normal form $h=[\hat{p}, \epsilon, \hat{r}, 0]$ with $\epsilon=\operatorname{sgn} q^{o}$ and $\hat{p}, \hat{r}$ depending on $N$, $\lambda_{1}$ and $u_{4}$ only.

Proof. See [14].

This theorem applies to cases $\mathbf{I}_{\mathbf{0}}$ to IV in Theorem $\mathbf{3} \cdot 2 \cdot 2$.

3.3.6. Proof of Theorem $3 \cdot 2 \cdot 2$. We follow the classical framework as described in the proof of Theorem $3 \cdot 2 \cdot 1$. We omit the details of the calculations.

For each given normal form $g$, we find an intrinsic submodule $R^{\prime}$ contained in the intrinsic part of the $\mathbf{D}_{4}$-unipotent tangent space of $g$ and from Proposition $2 \cdot 5 \cdot 2, R^{\prime}$ is contained in the set of higher order terms of $g$. Then we make the change of coordinates modulo $R^{\prime}$ for a $\mathbf{D}_{4}$-bifurcation problem $f=[p, q, r, s]$ establishing that $f$

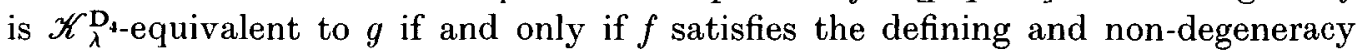
conditions stated in each case. Then we apply Corollary $2 \cdot 3 \cdot 2$ to get the required information about miniversal unfoldings.

Case $\mathrm{I}_{0}$ was proved in Theorem $3 \cdot 2 \cdot 1$.

For Case $\mathbf{I}_{1}$, take $g=\left[m N+\epsilon_{3} N \lambda_{1}+\epsilon_{4} \lambda_{1}^{2}, \epsilon_{5}, 1,0\right]$ where $\epsilon_{3}^{2}=\epsilon_{4}^{2}=\epsilon_{5}^{2}=1$ and $m \neq \pm 1,0$. Then

$$
\mathscr{T} \mathscr{U}^{\mathbf{D}_{4}}(g)=\left[\mathscr{M}_{u}^{3}+\left\langle N^{2}, \Delta, u_{4}\right\rangle, \mathscr{M}_{u}^{2}+\left\langle N, \Delta, u_{4}\right\rangle, \mathscr{M}_{u}, \mathscr{E}_{u}\right]+\left\langle\left[m N \lambda_{1}, \epsilon_{5} \lambda_{1}, 0,0\right]\right\rangle
$$

and

$$
\begin{aligned}
\mathscr{T}_{e}^{\mathbf{D}_{\lrcorner}(g)=} & \mathscr{T} \mathscr{U}^{\mathbf{D}_{\lrcorner}(g)} \\
& +\mathbb{R} .\left\{\left[m N, \epsilon_{5}, 0,0\right],\left[\lambda_{1}^{2}, 0,0,0\right],\left[\epsilon_{3} N+2 \epsilon_{4} \lambda_{1}, 0,0,0\right],\left[N \lambda_{1}, 0,0,0\right],[0,0,1,0]\right\} .
\end{aligned}
$$




\section{JacQues-Élie Furter, Angela Maria Sitta and Ian Stewart}

As a consequence, $g$ has $\mathbf{D}_{4}$-co-dimension 2 and topological co-dimension 1 since $m$ is a modal parameter and $[1,0,0,0]$ is also an unfolding term.

Now, let

$$
R^{\prime}=\left[\mathscr{M}_{u}^{3}+\mathscr{M}_{(N, \Delta)}^{2}+\left\langle\Delta, u_{4}\right\rangle, \mathscr{M}_{(N, \Delta)}+\mathscr{M}_{\left(\lambda_{1}, u_{4}\right)}^{2}+\left\langle u_{4}\right\rangle, \mathscr{M}_{u}, \mathscr{E}_{u}\right]
$$

From Lemma $3 \cdot 3 \cdot 1, R^{\prime}$ is intrinsic.

For Case $\mathbf{I}_{2}$, take $g=\left[m N+n N u_{4}+\epsilon_{0} \lambda_{1}, \epsilon_{5}, \lambda_{1}+\epsilon_{8} u_{4}, 0\right]$ where $m \neq \pm 1,0$, $\epsilon_{0}^{2}=\epsilon_{5}^{2}=\epsilon_{8}^{2}=1$ and $n \neq 0$. Then

$$
\begin{aligned}
\mathscr{T} \mathscr{U}^{\mathrm{D}_{4}}(g)= & {\left[\mathscr{M}_{u}^{3}+\left\langle N^{2}, N \lambda_{1}, \Delta\right\rangle+\mathscr{M}_{\left(\lambda_{1}, u_{4}\right)}^{2}, \mathscr{M}_{u}^{2}+\left\langle N, \Delta, \lambda_{1}\right\rangle, \mathscr{M}_{u}^{2}+\langle\Delta\rangle, \mathscr{M}_{u}\right] } \\
& +\left\langle\left[m N u_{4}, \epsilon_{5} u_{4}, 0,0\right],\left[0,0, N, \epsilon_{5} m\right],\right. \\
& {\left.\left[0,0, \epsilon_{0} \lambda_{1}, \epsilon_{5}\left(1-m^{2}\right)\right],\left[\epsilon_{0} u_{4}, 0, u_{4}, 0\right]\right\rangle, } \\
\mathscr{T}_{e}^{\mathbf{D}_{4}}(g)= & \mathscr{T} \mathscr{U}^{\mathbf{D}_{4}}(g)+\mathbb{R} \cdot\left\{\left[m N+n N u_{4}, \epsilon_{5}, 0,0\right],\left[\epsilon_{0}, 0,1,0\right],\left[\epsilon_{0} \lambda_{1}, 0, \lambda_{1}, 0\right],\right. \\
& {\left.\left[2 n N u_{4}, 0, \lambda_{1}, 0\right],\left[0,0, u_{4}, 0\right]\right\} . }
\end{aligned}
$$

The unfolding terms are $[N, 0,0,0],\left[N u_{4}, 0,0,0\right]$, and $[0,0,1,0]$. It follows that $g$ has $\mathbf{D}_{4}$-co-dimension 3 and topological co-dimension 1 since $m$ and $n$ are modal parameters.

In this case $R^{\prime}=\left[\mathscr{M}_{u}^{3}+\mathscr{M}_{u} \cdot\langle\Delta\rangle+\mathscr{M}_{\left(\lambda_{1}, u_{4}\right)}^{2}, \mathscr{M}_{u}^{2}, \mathscr{M}_{u}^{2}, \mathscr{M}_{u}\right]$.

For Case II, take $g=\left[\epsilon_{2} N^{2}+\epsilon_{0} \lambda_{1}, \epsilon_{5}, 1,0\right]$ where $\epsilon_{0}^{2}=\epsilon_{2}^{2}=\epsilon_{5}^{2}=1$. Then

$$
\mathscr{T} \mathscr{U}^{\mathbf{D}_{4}}(g)=\left[\mathscr{M}_{u}^{3}+\left\langle N \lambda_{1}, \lambda_{1}^{2}, \Delta, u_{4}\right\rangle, \mathscr{M}_{u}, \mathscr{M}_{u}, \mathscr{E}_{u}\right]
$$

As $\quad \mathscr{T}_{\boldsymbol{D}^{4}}(g)=\mathscr{T} \mathscr{U}^{\mathrm{D}_{4}}(g)+\mathbb{R} .\left\{\left[N^{2}, 0,0,0\right],[1,0,0,0],\left[\lambda_{1}, 0,0,0\right],[0,1,0,0],[0,0,1,0]\right\}$, the unfolding term is $[N, 0,0,0]$ and by definition $g$ has $\mathbf{D}_{4}$ and topological codimension equal to 1 .

In this case $R^{\prime}=\left[\mathscr{M}_{u}^{3}+\left\langle N \lambda_{1}, \lambda_{1}^{2}, \Delta, u_{4}\right\rangle, \mathscr{M}_{u}, \mathscr{M}_{u}, \mathscr{E}_{u}\right]$.

For Case III, take $g=\left[-\epsilon_{5} N+\epsilon_{0} \lambda_{1}, \epsilon_{5}, 1+\epsilon_{7} N, 0\right]$ where $\epsilon_{0}^{2}=\epsilon_{5}^{2}=\epsilon_{7}^{2}=1$. Then

$$
\begin{aligned}
\mathscr{T} \mathscr{U}^{\mathrm{D}_{4}}(g)= & {\left[\mathscr{M}_{u}^{3}+\left\langle\Delta^{2}, u_{4}, \lambda_{1}^{2}, N \Delta\right\rangle, \mathscr{M}_{u}^{2}+\left\langle\Delta, u_{4}\right\rangle, \mathscr{M}_{u}^{2}+\left\langle\Delta, \lambda_{1}, u_{4}\right\rangle, \mathscr{M}_{u}\right] } \\
& +\left\langle\left[N^{2},-N, 0,0\right],\left[\epsilon_{0} N \lambda_{1}, 0, N, 0\right],[\Delta,-N, 0,0],\left[-N \lambda_{1}, \lambda_{1}, 0,0\right],\right. \\
& {\left.\left[\epsilon_{0} N \lambda_{1}-\epsilon_{5} N^{2}, 0,0,0\right],[0,0,-N, 1]\right\rangle . }
\end{aligned}
$$

As

$$
\mathscr{T}_{\boldsymbol{e}}^{\mathbf{D}_{\mathbf{4}}}(g)=\mathscr{T} \mathscr{U}^{\mathbf{D}_{4}}(g)+\mathbb{R} .\left\{[-N, 1,0,0],[1,0,0,0],\left[\lambda_{1}, 0,0,0\right],[0,0, N, 0],[0,0,1,0]\right\},
$$

we take $[N, 0,0,0]$ as the unfolding term. So, $g$ has both smooth and topological $\mathbf{D}_{4^{-}}$ co-dimensions equal to 1 .

Here $R^{\prime}=\left[\mathscr{M}_{u}^{3}+\mathscr{M}_{u} \cdot\langle\Delta\rangle+\left\langle\lambda_{1}^{2}, u_{4}\right\rangle, \mathscr{M}_{u}^{2}+\left\langle\Delta, u_{4}\right\rangle, \mathscr{M}_{u}^{2}+\left\langle\Delta, \lambda_{1}, u_{4}\right\rangle, \mathscr{M}_{u}\right]$.

For Case IV, take $g=\left[\epsilon_{1} N+\epsilon_{9} N^{2}+\epsilon_{0} \lambda_{1}, \epsilon_{1}+m_{2} \lambda_{1}, 1,0\right]$ where $\epsilon_{0}^{2}=\epsilon_{1}^{2}=\epsilon_{9}^{2}=1$ and $m_{2} \neq 0$. Then,

$$
\begin{aligned}
\mathscr{T} \mathscr{U}^{\mathrm{D}_{4}}(g)= & {\left[\mathscr{M}_{u}^{3}+\left\langle u_{4}, \lambda_{1}^{2}, \Delta^{2}, \Delta \lambda_{1}, \Delta N\right\rangle, \mathscr{M}_{u}^{2}+\left\langle\Delta, u_{4}\right\rangle, \mathscr{M}_{u}^{2}+\left\langle\Delta, \lambda_{1}, u_{4}\right\rangle, \mathscr{M}_{u}\right] } \\
+ & \left\langle\left[N \lambda_{1}, \lambda_{1}, 0,0\right],\left[N^{2}, N, 0,0\right],\right. \\
& {\left.\left[N^{2}-\Delta, 0,0,0\right],\left[0, \epsilon_{0} \lambda_{1}, 0,1\right],[0,0, N, 1]\right\rangle . } \\
\mathscr{T T}_{e}^{\mathrm{D}_{4}}(g)= & \mathscr{T} \mathscr{U}^{\mathrm{D}_{4}}(g)+\mathbb{R} .\left\{\left[\epsilon_{1} N+\epsilon_{9} N^{2}, \epsilon_{1}, 0,0\right],\left[\epsilon_{9} N^{2}-\epsilon_{0} \lambda_{1}, 0,0,0\right],\left[\epsilon_{0}, m_{2}, 0,0\right],\right. \\
& {\left.\left[\epsilon_{0} \lambda_{1}, m_{2} \lambda_{1}, 0,0\right],[0,0,1,0]\right\} . }
\end{aligned}
$$


We choose $[N, 0,0,0]$ and $\left[0, \lambda_{1}, 0,0\right]$ as unfolding terms and so $g$ has $\mathbf{D}_{4}$-co-dimension 2 and topological co-dimension 1 since $m_{2}$ is a modal parameter.

Here $R^{\prime}=\left[\mathscr{M}_{u}^{3}+\mathscr{M}_{u} \cdot\langle\Delta\rangle+\left\langle\lambda_{1}^{2}, u_{4}\right\rangle, \mathscr{M}_{u}^{2}+\left\langle\Delta, u_{4}\right\rangle, \mathscr{M}_{u}^{2}+\left\langle\Delta, \lambda_{1}, u_{4}\right\rangle, \mathscr{M}_{u}\right]$.

For Case V, take $g=\left[\epsilon_{1} N+\epsilon_{0} \lambda_{1}, \epsilon_{6} \Delta+m_{1} \lambda_{1}, 1,0\right]$ where $\epsilon_{0}^{2}=\epsilon_{1}^{2}=\epsilon_{6}^{2}=1$ and $m_{1} \neq 0$. Then,

$$
\begin{gathered}
\mathscr{T} \mathscr{U}^{\mathrm{D}_{4}}(g)=\left[\mathscr{M}_{u}^{2}+\left\langle\Delta, u_{4}\right\rangle, \mathscr{M}_{u}^{2}+\left\langle\left[0, \epsilon_{1} N+\epsilon_{0} \lambda_{1}, 0,0\right], u_{4}\right\rangle, \mathscr{M}_{u}, \mathscr{E}_{u}\right], \\
\mathscr{T}_{e^{\mathrm{D}_{4}}}(g)=\mathscr{T} \mathscr{U}^{\mathrm{D}_{4}}(g)+\mathbb{R} .\left\{\left[\epsilon_{1} N, \epsilon_{6} \Delta, 0,0\right],\left[0,2 \epsilon_{6} \Delta+m_{1} \lambda_{1}, 0,0\right],\left[\epsilon_{0}, m_{1}, 0,0\right],\right. \\
\left.\quad\left[\epsilon_{0} \lambda_{1}, m_{1} \lambda_{1}, 0,0\right],[0,0,1,0]\right\} .
\end{gathered}
$$

Let $\left[0, \lambda_{1}, 0,0\right]$ and $[0,1,0,0]$ be the unfolding terms. So, $g$ has $\mathbf{D}_{4}$-co-dimension 2 and topological co-dimension 1 since $m_{1}$ is a modal parameter.

Here $R^{\prime}=\left[\mathscr{M}_{u}^{2}+\left\langle u_{4}\right\rangle, \mathscr{M}_{u}^{2}+\left\langle u_{4}\right\rangle, \mathscr{M}_{u}, \mathscr{E}_{u}\right]$.

\section{3·4. Remarks}

3.4.1. One-parameter problems. By setting $\lambda_{2}=0$ we can return to the problem of classifying $\mathbf{D}_{4}$-equivariant problems with one bifurcation parameter $\lambda_{1}$ and trivial action on it, which has been tackled in [6]. The choice of normal forms in that paper appear to be relatively random. Our additional criteria can be used to organize that list. For instance, the equivalent of Theorem $3 \cdot 3 \cdot 6$ on pre-normal forms applies. The classification is organized into three broad forms: $p_{N}^{o} \neq q^{o}(\neq 0), p_{N}^{o}=q^{o}(\neq 0)$ and $q^{o}=0$. When $q^{o} \neq 0$ and $p_{N}^{o} \neq q^{o}$, the normal form is of the type $(p(N, \lambda), \epsilon)$ where $\epsilon=\operatorname{sgn} q^{\circ}$ with the extended tangent space equal to

$$
\left(\left\langle N p_{N}-p, N p\right\rangle, 0\right)+\langle(\Delta, 0),(p, \epsilon)\rangle+\mathscr{E}_{\lambda} \cdot\left\langle\left(p_{\lambda}, 0\right)\right\rangle .
$$

This shows that we can reduce the problem to the discussion of

$$
\mathscr{I}=\left\langle N p_{N}-p, N p\right\rangle+\mathscr{E}_{\lambda} \cdot\left\langle p_{\lambda}\right\rangle
$$

in $\mathscr{E}_{(x, \lambda)}^{Z_{2}}$ and its equivalent for the higher order terms. By inspection, although $p$ is in $\mathbf{Z}_{2}$-equivariant form, $\mathscr{I}$ is of higher co-dimension than the $\mathbf{Z}_{2}$-extended tangent space for $p$ (equal to $\left\langle N p_{N}, p\right\rangle+\mathscr{E}_{\lambda} \cdot\left\langle p_{\lambda}\right\rangle$ ). It is clear that both codimensions are equal if and only if $p \in \mathscr{I}$. But by inspection this never happens.

When $p_{N}^{o}=q^{o}(\neq 0)$, we conjecture that normal forms are given by $(\epsilon N+p(N, \lambda), \epsilon)$ where $(p, \epsilon)$ is one of the previous normal forms when $p_{N}^{o}=0$ (with $p$ of order two in $N$ ).

When we restrict our normal forms to the subspace $\lambda_{2}=0$, we find those one parameter normal forms. The Case III (when $p^{o}=-q^{o}$ ) is of particular interest

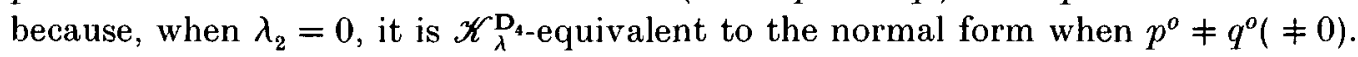
But with the additional parameter $\lambda_{2}$ we have to distinguish those two cases.

$3 \cdot 4 \cdot 2$. Variational problems. Another criterion for a normal form arises from the gradient structure of some bifurcation problems. For instance the first example in Section 5 of buckling of elastic shells usually has a variational formulation. We refer to Bridges and Furter [1] for a theory of contact equivalence of gradient bifurcation problems, remarking only that the idea is to seek normal forms and (if possible) universal unfoldings that are gradients of equivariant functionals. In our setting a routine calculation shows that

$$
f(z, \lambda)=p(u) z+q(u) \delta \bar{z}+r(u) \lambda_{2} \bar{z}+s(u) \lambda_{2} \delta z
$$




\section{Jacques-Élie Furter, Angela Maria Sitta and Ian Stewart}

is a gradient if and only if $q_{N}+2 p_{\Delta} \equiv 0$ and $r_{N}+s+2 \Delta s_{\Delta} \equiv 0$. By inspection the only case in Theorem $3 \cdot 2 \cdot 2$ that needs special investigation is Case III. All the other functions, as well as their miniversal unfoldings, are gradients. The normal form for Case III was chosen so that the germ is similar to case $\mathbf{I}_{0}$ when $\lambda_{2}=0$. We can abandon that requirement and obtain the following gradient normal form instead:

$$
\text { Case III': }\left[\epsilon_{0} \lambda_{1}-\epsilon_{5} N+\epsilon_{10} N^{2}+\alpha N, \epsilon_{5}, 1,0\right] \text {, }
$$

where $\epsilon_{10}=-\operatorname{sgn} p_{N}^{o} \cdot r^{o} \cdot\left(p_{N \lambda_{1}}^{o}+q_{\lambda_{1}}^{o}\right)$. Thus, we have the following result.

THeOREM 3·4:1. The list of gradient bifurcation problems in $\overrightarrow{\mathscr{E}}_{(z, \lambda)}$ up to gradient topological co-dimension 1 consists of the Cases $\mathbf{I}$ to $\mathrm{V}$ in Theorem 3.2.2 with Case III

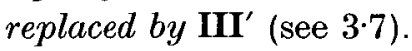

This is again a situation where symmetry puts enough constraints on the diagrams so that the difference between gradient and non-gradient systems is negligible, at least in low co-dimension. This also happens for $\mathbf{D}_{n}$-equivariant $(n \geqslant 3)$ bifurcation problems with only one parameter, see Bridges and Furter[1].

\section{Bifurcation diagrams}

A geometrical description of a normal form for a bifurcation problem is given by its bifureation diagram, that is, its zero-set. We draw schematic bifurcation diagrams for the $\mathbf{D}_{\mathbf{4}}$-problems and their corresponding universal unfoldings according to the regions of $\lambda$-space where the solutions are defined. Recall that with two parameters contact equivalence preserves the 'organization' only of the open regions of the $\lambda$ plane that correspond to different characteristics of the zero-set (such as number of solutions, stability, and so on). However, in our situation the $\lambda_{1}$-axis is invariant, which adds to the structure. For the pictures we use a polar coordinate representation via the parametrisation $\left(\lambda_{1}, \lambda_{2}\right)=(R \cos s, R \sin s)$ with $R>0$. In some cases, the diagram changes when $R$ varies. The asymptotic orbital stability of the solutions, determined by the eigenvalues of the Jacobian, is also shown. Although stability is not in general invariant under contact equivalence there are many cases when it is - a feature that we discuss below when it arises.

\subsection{Isotropy types for $D_{4}$-problems}

When $\Gamma$ acts trivially on the parameter space $\mathbb{R}^{l}$, the isotropy subgroup $\Sigma_{(x, \lambda)}$ of $(x, \lambda) \in \mathbb{R}^{n+\ell}$, given by $\Sigma_{(x, \lambda)}=\{\gamma \in \Gamma \mid \gamma \cdot x=x\}$, is independent of $\lambda$. The fixed-point subspace for a subgroup $\Sigma$ of $\Gamma$ is the following subspace of $\mathbb{R}^{n+\ell}$ :

$$
\operatorname{Fix}(\Sigma)=\left\{x \in \mathbb{R}^{n} \mid \sigma . x=x, \forall \sigma \in \Sigma\right\} \times \mathbb{R}^{\prime} .
$$

In our situation, however, we must take into account the action of $\Gamma$ on $\mathbb{R}^{l}$. In that case it is easy to show the following, remembering that the actions $\gamma_{n}, \gamma_{\ell}$ of $\gamma \in \Gamma$ on $\mathbb{R}^{n}$ and $\mathbb{R}^{l}$ are in general not equal:

Lemma 4·1. Under the previous hypotheses,

$$
\Sigma_{(x, \lambda)}=\Sigma_{x} \cap \Sigma_{\lambda}
$$

and

$$
\operatorname{Fix}_{\mathbb{R}^{n+\ell}}(\Sigma)=\operatorname{Fix}_{\mathbf{R}^{n}}(\Sigma) \times \operatorname{Fix}_{\mathbf{R}^{\prime}}(\Sigma) .
$$


Table 2. Fixed-point data

\begin{tabular}{ll}
\hline \multicolumn{1}{c}{$\Sigma$} & \multicolumn{1}{c}{ Fix $(\Sigma)$} \\
\hline $\mathbf{D}_{4}$ & $x_{1}=x_{2}=0$ \\
$Z_{2}(\kappa)$ & $x_{2}=0$ \\
$Z_{2}^{c}(\kappa)$ & $x_{1}=0$ \\
$Z_{2}(i \kappa)$ & $x_{1}=x_{2}, \lambda_{2}=0$ \\
$Z_{2}^{c}(i \kappa)$ & $x_{1}=-x_{2}, \lambda_{2}=0$ \\
1 & $\mathbb{R}^{4}$ \\
\hline
\end{tabular}

For our situation, the trivial solution $(0,0) \in \mathbb{R}^{4}$ is of maximal isotropy $\mathbf{D}_{\mathbf{4}}$. On the state space $\mathbb{R}^{2}$ there are 4 fixed-point subspaces of dimension 1 with isotropy subgroups isomorphic to $\mathrm{Z}_{2}: x_{2}=0\left(\mathrm{Z}_{2}(\kappa)\right), x_{1}=0\left(\mathrm{Z}_{2}(-\kappa)\right), x_{1}=x_{2}\left(\mathrm{Z}_{2}(i \kappa)\right)$ and $x_{1}=-x_{2}\left(\mathbf{Z}_{2}(-i \kappa)\right)$, where the pairs $\mathbf{Z}_{2}(\kappa), \mathbf{Z}_{2}^{c}(\kappa)$ and $\mathbf{Z}_{2}(i \kappa), \mathbf{Z}_{2}^{c}(i \kappa)$ are conjugate. The corresponding action on the $\lambda$-space is obtained by applying the group homomorphism $i \mapsto \kappa, \kappa \mapsto \mathbf{1}$ to $\mathbf{D}_{4}$, hence it is equal to $\mathbf{Z}_{2}(\kappa)$ for $\mathbf{Z}_{2}^{(c)}(i \kappa)$ and $\mathbf{1}$ for $Z_{2}^{(c)}(\kappa)$. This means that the isotropy subgroups and their fixed-point subspaces for the chosen $D_{4}$-action are as listed in Table 2 above.

Note that $(y, 0) \in \operatorname{Fix}\left(\mathbf{Z}_{2}(\kappa)\right)$ at $\left(\lambda_{1}, \lambda_{2}\right)$ if and only if $(0, y) \in \operatorname{Fix}\left(\mathbf{Z}_{2}^{c}(\kappa)\right)$ at $\left(\lambda_{1},-\lambda_{2}\right)$. The space Fix (1) is globally invariant under the symmetry $\lambda_{2} \mapsto-\lambda_{2}$, so it is enough to consider $\lambda_{2} \geqslant 0$.

\section{4·2. Linearized stability}

Our linear stability information is given for the system

$$
\frac{d x}{d t}+g(x, \lambda)=0
$$

that is, asymptotic stability is given by positive eigenvalues. Modulo the usual restrictions (Vanderbauwhede[16]) they are also valid when $g=0$ is a bifurcation equation obtained via Liapunov-Schmidt reduction.

The matrix $g_{x}$ of the linearization of $g$ is diagonal on the fixed-point subspaces with

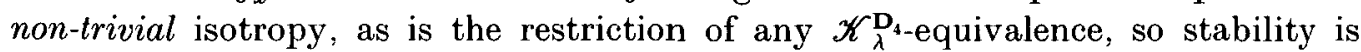
preserved for solutions with non-trivial isotropy. Moreover, the determinant of the linearization on Fix (1) is also preserved (as for any contact equivalence).

The general form for a $\mathbf{D}_{4}$-problem is given by

$$
g(z, \lambda)=p(u) z+q(u) \delta \bar{z}+r(u) \lambda_{2} \bar{z}+s(u) \lambda_{2} \delta z
$$

where $u=\left(N, \Delta, \lambda_{1}, u_{4}\right)$. The full solution of $g=0$ and the eigenvalue data are listed in Table 3.

General formulas for the determinant and trace of the linearization of $g$ are given by $\left|g_{x}\right|=\left|g_{z}\right|-\left|g_{z}\right|$ and $\operatorname{tr} g_{x}=2$ re $g_{z}$, where $g$ is considered as a function of two independent variables $z, \bar{z}$. The trace and determinant of the linearization on Fix (1) are:

$$
\begin{aligned}
\operatorname{tr} g_{x}=N p_{N} & +2 \Delta p_{\Delta}-N q-\Delta q_{N}-2 \Delta N q_{\Delta}+\delta \lambda_{2}\left(-r_{N}-2 N r_{\Delta}+s+N s_{N}+2 \Delta s_{\Delta}\right) \\
\operatorname{det} g_{x}=-4 q p_{N} & +8 \Delta\left(p_{\Delta} q_{N}-p_{N} q_{\Delta}\right)+\delta \lambda_{2}\left[8\left(p_{\Delta} r_{N}-p_{N} r_{\Delta}\right)\right. \\
& \left.+4\left(s q_{N}-q s_{N}\right)+8 \Delta\left(s_{\Delta} q_{N}-q_{\Delta} s_{N}\right)\right]+u_{4}\left[4 s r_{N}+8 \Delta\left(s_{\Delta} r_{N}-s_{N} r_{\Delta}\right)\right] .
\end{aligned}
$$


Table 3. Solutions and stability for $\mathbf{D}_{4}$-problems

\begin{tabular}{|c|c|c|}
\hline $\begin{array}{c}\text { Fixed-point } \\
\text { subspaces }\end{array}$ & Solutions & Eigenvalues \\
\hline $\begin{array}{l}\text { Fix }\left(\mathbf{D}_{4}\right) \\
\operatorname{Fix}\left(Z_{2}(\kappa)\right)\end{array}$ & $\begin{array}{l}x_{1}=x_{2}=0 \\
x_{2}=0 \\
p=q x_{1}^{2}-\lambda_{2} r+s \lambda_{2} x_{1}^{2}\end{array}$ & $\begin{array}{l}p+\lambda_{2} r \quad \text { and } p-\lambda_{2} r \\
\left(p_{N}-q+\left(2 p_{\Delta}-q_{N}\right) x_{1}^{2}-2 q_{\Delta} x_{1}^{4}\right) \\
\quad+\lambda_{2}\left(r_{N}-s+\left(2 r_{\Delta}-s_{N}\right) x_{1}^{2}-2 s_{\Delta} x_{1}^{4}\right) \\
\quad \text { and }\left(q x_{1}^{2}-\lambda_{2} r\right)\end{array}$ \\
\hline $\operatorname{Fix}\left(\mathrm{Z}_{2}(-\kappa)\right)$ & $\begin{array}{l}x_{1}=0 \\
p=q x_{2}^{2}+\lambda_{2} r-s \lambda_{2} x_{2}^{2}\end{array}$ & $\begin{array}{l}\left(p_{N}-q+\left(2 p_{\Delta}-q_{N}\right) x_{2}^{2}-2 q_{\Delta} x_{2}^{4}\right) \\
\quad+\lambda_{2}\left(-r_{N}+s-\left(2 r_{\Delta}-s_{N}\right) x_{2}^{2}+2 s_{\Delta} x_{2}^{4}\right) \\
\quad \text { and }\left(q x_{2}^{2}+\lambda_{2} r\right)\end{array}$ \\
\hline $\operatorname{Fix}\left(\mathbf{Z}_{2}^{(c)}(i \kappa)\right)$ & $\begin{array}{l}x_{1}= \pm x_{2} \text { and } \quad \lambda_{2}=0 \\
p=0\end{array}$ & $p_{N}$ and $-q$ \\
\hline $\begin{array}{l}\text { Fix (1) } \\
\lambda_{2} r+\delta q=0\end{array}$ & $p+s \delta \lambda_{2}=0$ & Trace and determinant given below in $(4 \cdot 1)$ \\
\hline
\end{tabular}

Note that solutions in Fix $\left(Z^{(c)}(i \kappa)\right)$ are always contained in $\operatorname{Fix}(\mathbf{1})$.

Because of the non-trivial action of $\Gamma$ on $\mathbb{R}^{l}$, the linearization satisfies

$$
\gamma_{n}^{-1} g_{x}\left(\gamma_{n} x, \gamma_{\ell} \lambda\right) \gamma_{n}=g_{x}(x, \lambda) \quad \forall \gamma \in \Gamma .
$$

When $\gamma_{\ell}$ is trivial this means that the linearizations are conjugate along the group orbits (for $\lambda$ fixed), that is, the stability is constant along them. Hence (4.2) also means that stability is constant along the group orbits, but this time this also involves the $\lambda$-component.

Note also that because the actions of $\mathbf{D}_{4}$ on the fixed-points subspaces of the nontrivial isotropy subgroups are absolutely irreducible there cannot be a Hopf bifurcation from points in those subspaces. So periodic orbits can be born only through tertiary bifurcation inside Fix (1).

\section{4:3. Diagrams for the generic normal form}

We now study the bifurcation diagrams for the generic normal form, namely

$$
g\left(z, \lambda_{1}, \lambda_{2}\right)=\left(m N+\epsilon_{0} \lambda_{1}\right) z+\epsilon_{5} \delta \bar{z}+\lambda_{2} \bar{z}
$$

with $m \neq \pm 1,0$ and $\epsilon_{0}= \pm 1, \epsilon_{5}= \pm 1$. It is enough to consider $\epsilon_{0}=-1$. Moreover, it is possible to show (see [4]) that (4.3) is also the generic normal form for the more restrictive contact equivalence where $\lambda_{1}$ and $\lambda_{2}$ are not mixed but $\lambda_{1}$ is of higher status. This is represented in the change of coordinates by

$$
\Lambda\left(\lambda_{1}, \lambda_{2}\right)=\left(\Lambda_{1}\left(\lambda_{1}, \lambda_{2}\right), \Lambda_{2}\left(\lambda_{2}\right)\right) .
$$

It means that the $\lambda_{2}$-sequence of $\lambda_{1}$-slices is an invariant of contact equivalence.

In cartesian coordinates $\left(x_{1}, x_{2}, \lambda_{1}, \lambda_{2}\right)$ the miniversal unfolding $G$ of $g$ takes the form

$$
G\left(x_{1}, x_{2}, \lambda_{1}, \lambda_{2}, \beta\right)=\left(\begin{array}{c}
\left(-\lambda_{1}+\lambda_{2}\right) x_{1}+\left(\hat{m}-\epsilon_{5}\right) x_{1}^{3}+\left(\hat{m}+\epsilon_{5}\right) x_{1} x_{2}^{2} \\
-\left(\lambda_{1}+\lambda_{2}\right) x_{2}+\left(\hat{m}-\epsilon_{5}\right) x_{2}^{3}+\left(\hat{m}+\epsilon_{5}\right) x_{1}^{2} x_{2}
\end{array}\right),
$$

where $\hat{m}=m+\beta$. Recall that $m$ is a modal parameter, that is, small changes in $\beta$ have no topological influence on the bifurcation diagrams (except perhaps for isolated 'exceptional' values of $m$ ). We analyse each case according to the variation of $m$. 
Table 4. Solutions and their stability for the generic normal form

\begin{tabular}{|c|c|c|c|}
\hline $\begin{array}{l}\text { Isotropy } \\
\text { subgroup }\end{array}$ & $\begin{array}{l}\text { Regions of the } \\
\left(\lambda_{1}, \lambda_{2}\right) \text {-plane }\end{array}$ & Solutions & Eigenalues \\
\hline $\operatorname{Fix}\left(Z_{2}(\kappa)\right)$ & $\left(m-\epsilon_{5}\right)\left(\lambda_{1}-\lambda_{2}\right)>0$ & $\begin{aligned} x_{2} & =0 \\
x_{1}^{2} & =\frac{\left(\lambda_{1}-\lambda_{2}\right)}{\left(m-\epsilon_{5}\right)}\end{aligned}$ & $\begin{array}{l}m-\epsilon_{5} \\
\frac{\epsilon_{5} \lambda_{1}-m \lambda_{2}}{m-\epsilon_{5}}\end{array}$ \\
\hline $\operatorname{Fix}\left(\mathbf{Z}_{2}^{c}(\kappa)\right)$ & $\left(m-\epsilon_{5}\right)\left(\lambda_{1}+\lambda_{2}\right)>0$ & $\begin{aligned} x_{1} & =0 \\
x_{2}^{2} & =\frac{\left(\lambda_{1}+\lambda_{2}\right)}{\left(m-\epsilon_{5}\right)}\end{aligned}$ & $\begin{array}{l}m-\epsilon_{5} \\
\frac{\epsilon_{5} \lambda_{1}+m \lambda_{2}}{m-\epsilon_{5}}\end{array}$ \\
\hline $\operatorname{Fix}\left(\mathbf{Z}_{2}^{(c)}(i \kappa)\right)$ & $\begin{array}{l}m \lambda_{1}>0 \\
\lambda_{2}=0\end{array}$ & $\begin{array}{l}x_{1}= \pm x_{2} \\
x_{1}^{2}=\frac{1}{2 m} \lambda_{1}\end{array}$ & $m$ and $-\epsilon_{5}$ \\
\hline \multirow[t]{2}{*}{ Fix (1) } & $m \lambda_{1}>0$ & $x_{1}^{2}=\frac{1}{2}\left(\frac{\lambda_{1}}{m}+\epsilon_{5} \lambda_{2}\right)$ & $\operatorname{sg} \operatorname{tr}\left(g_{x}\right)=\operatorname{sg}\left(m-\epsilon_{5}\right)$ \\
\hline & $\left|\lambda_{2}\right|<\frac{1}{m} \lambda_{1}$ & $x_{2}^{2}=\frac{1}{2}\left(\frac{\lambda_{1}}{m}-\epsilon_{5} \lambda_{2}\right)$ & $\operatorname{sg} \operatorname{det}\left(g_{x}\right)=\operatorname{sg}\left(-\epsilon_{5} m\right)$ \\
\hline
\end{tabular}

The trivial solution exists for $\lambda \in \mathbb{R}^{2}$ and its stability is given by the signs of the eigenvalues, which are $-\lambda_{1}-\lambda_{2},-\lambda_{1}+\lambda_{2}$. We have chosen $\epsilon_{0}$ such that for $\lambda_{1}$ large negative ( $\lambda_{2}$ small) then the trivial solution is stable. It looses stability when we cross the lines $\lambda_{1} \pm \lambda_{2}=0$, with a bifurcation to solutions in Fix $\left(Z_{2}^{(c)} \kappa\right)$. The rest of the solutions are given in Table 4 .

One way of representing the bifurcation diagrams is to consider first the $\lambda$-plane and to describe the boundaries of the regions where the solution set does not change qualitatively. Note that there is an exchange principle for the stability of non-trivial solutions, that is, the solutions in Fix (1) and in Fix $\left(Z_{2}^{(c)}(\kappa)\right)$ cannot be stable at the same time. So we consider two situations, depending on whether the solutions in Fix (1) are stable or not.

We consider the representation of the diagrams by a parametrization in polar coordinates: $\left(\lambda_{1}, \lambda_{2}\right)=(R \cos s, R \sin s)$ with $R>0$. The solution branches are functions of the bifurcation parameters. They are drawn in the space $\left(s, x_{1}, x_{2}\right)$ with axes oriented as shown in the figure. In the generic case the qualitative behaviour of the diagrams does not depend on the values of the radius $R$, so we have 'factored out' the variable $R$.

First, let $\epsilon_{5}=1$ with $m>1$. Then the solutions in Fix (1) are unstable. What actually happens is that as $\lambda_{1}$ grows from $-\infty$ the trivial solution goes unstable, bifurcating to stable $\mathbf{Z}_{2}^{(c)}(\kappa)$-solutions (which group occurs depends on the sign of $\lambda_{2}$ ) which then go unstable when solutions with trivial symmetry bifurcate. Secondly, if $\epsilon_{5}=-1$ and $m>1$, the solutions with trivial symmetry are now stable. They come about from secondary bifurcation destabilizing solutions in $\operatorname{Fix}\left(\mathbf{Z}_{2}^{(c)}(\kappa)\right)$.

Note that when $m>1$ and $\epsilon_{5}=1$ we have the intersection of two 'pitchforks' in the $\left(x_{1}, s\right)$-plane. The same occurs in the $\left(s, x_{2}\right)$-plane. In the regions of the $\lambda$-plane defined by $\lambda_{1}>0$ and $\lambda_{2}+\lambda_{1} / m>0$, or $\lambda_{1}>0$ and $\lambda_{2}-\lambda_{1} / m<0$, the branches in the space $\left(s, x_{1}, x_{2}\right)$ have the property that for each fixed $s$ there are four points as solutions. The contact point of these branches with the 'pitchforks' are those 


\section{Jacques-Élie Furter, Angela Maria Sitta and Ian Stewart}

corresponding with the coincidence of the solutions which arise from the fixed-point subspaces, as described previously.

To draw the bifurcation diagrams we analyse the number of solutions for the bifurcation problem defined by the generic normal form for each fixed $\left(\lambda_{1}, \lambda_{2}\right)$. Schematic representations of these solutions are shown in Figs. 3 and 4. The corresponding bifurcation diagrams are drawn in Figs. 1 and 2 respectively.

\section{Sample applications}

In this section we describe two problems where the theory that we have developed can be applied. We concentrate on showing that the mathematical models lead to bifurcation equations which are $\mathbf{D}_{4}$-equivariant of the type studied in Sections 3 and 4.

The main requirement is a linearization with two independent but interchangeable modes. Nonlinear effects can then be taken care of via the classification of contact equivalent bifurcation problems in $\overrightarrow{\mathscr{E}}_{(z, \lambda)}$ (provided the model leads to a problem of finite co-dimension). The detailed study of the full nonlinear symmetry is postponed to a future paper. In broad terms, provided no additional symmetry has been forgotten or introduced in the model, and if there are $k$ perturbation parameters (in addition to the two bifurcation parameters), then we expect the bifurcation problem to be of co-dimension $k$ and we expect to encounter singularities that arise in the universal deformations of co-dimension $k$ problems.

\subsection{Buckling of a thin elastic shell}

In [8] there is a study of the buckling of a thin isotropic rectangular elastic plate subject to a compressive load $\lambda$, uniformly distributed over the end faces as shown in Figure 5.

A mathematical formulation of the plate problem is given by the nondimensionalized von Kármán equations for $w$, the vertical displacement, and $f$, the stress function:

$$
\left.\begin{array}{rl}
\Delta^{2} f & =-\frac{1}{2}[w, w], \\
\Delta^{2} w & =[w, f]+\lambda\left[w, f_{0}\right] .
\end{array}\right\}
$$

Here $w, f \in H_{o}^{2}(\Omega),[u, v]=u_{x x} v_{x x}-2 u_{x y} v_{x y}+u_{y y} v_{y y}$, and the appropriate remaining boundary conditions depend on how the plate is attached along its edges. The excess stress due to extra compression beyond the critical buckling load is given by $\lambda f_{0}$.

Generically, there is a one-dimensional kernel for the linearization of $(5 \cdot 1)$ at the critical load. However, depending on the aspect ratio of the plate, there are situations where two modes compete. Those modes represent two buckled configurations with consecutive wave numbers. When this happens (5-1) reduces to a $\mathbf{Z}_{2} \oplus \mathbf{Z}_{2}$-equivariant bifurcation problem with the bifurcation parameter being the load $\lambda$ (without symmetry acting on it). Note that in the case of a square plate the classical von Kármán equations $(5 \cdot 1)$ have only a one-dimensional kernel at the principal bifurcation; that is, there are no competing modes.

A general situation leading to our framework needs a two-dimensional kernel $\{(x, y)\}$ with two parameters $\left(\lambda_{1}, \lambda_{2}\right)$, with $x$ and $y$ being interchangeable when $\lambda_{2}=0$. This suggest studying the buckling of a thin anisotropic elastic square plate when a 


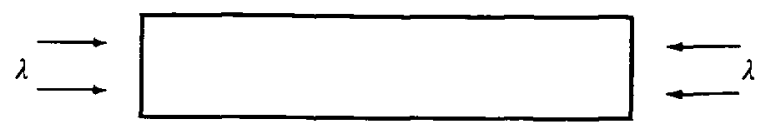

Fig. 5. Rectangular plate subject to lateral compressive load.

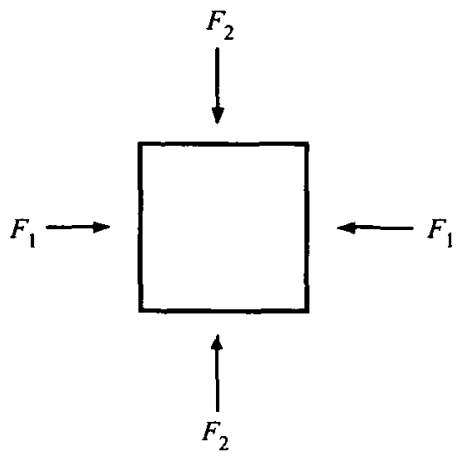

Fig. 6. Square plate subject to uniform compression.

pair of forces $F_{1}$ and $F_{2}$ act on the four edges as shown in Fig. 6. Our general theory can be applied to the bifurcation equations obtained from the von Kármán approximation to this problem. Those equations represent a $\mathbf{D}_{\mathbf{4}}$-equivariant problem with symmetry on the bifurcation parameters, given by the forces $\lambda_{1}=F_{1}$ and $\lambda_{2}=F_{2}-F_{1}$. But we still have a one-dimensional kernel at the bifurcation and so only a $Z_{2}$-action in space.

We can take care of the situation in two ways. We can consider the buckling of a thin elastic shell, or consider some anisotropic material (or non-uniform compressive load). In the first case we consider the shell as some buckled state of the original isotropic elastic square plate and look for secondary bifurcation with two competing modes. A fairly general formulation of the shallow shell equations is

$$
\begin{aligned}
\Delta^{2} f & =-\frac{1}{2}[w, w]-k_{y} w_{x x}-k_{x} w_{y y}, \\
\Delta^{2} w & =[w, f]+\lambda\left[w, f_{0}\right]+k_{y} f_{x x}+k_{x} f_{y y},
\end{aligned}
$$

where $k_{x}$ and $k_{y}$ represent the sectional curvatures of the shell and $\Delta$ is the Laplacian.

As a simple example of the buckling of a shell, assume the following for a shallow shell : the (non-negative) radii of curvature $R_{x}, R_{y}$ are roughly constant in the midregion of the surface, the projection of the shell is the unit square $[0,1] \times[0,1]$, and the shell is hinged at the boundary of the square (Dirichlet boundary conditions). We moreover assume that the equilibria correspond to states where the normal compressive stresses $\mathrm{N}_{x}=f_{x x}, \mathbf{N}_{y}=f_{y y}$ are constant with $f_{x y}=0$. Then the model reduces to the following 8 th order differential equation in $w$ :

$$
\Delta^{4} w+\left(k_{y}^{2} w_{x x x x}+2 k_{x} k_{y} w_{x x y y}+k_{y}^{2} w_{y y y}\right)+\lambda \Delta^{2}\left(\mathbf{N}_{x} w_{x x}+\mathbf{N}_{y} w_{y y}\right)=0
$$

with constants $k_{x}=1 / R_{x}$ and $k_{y}=1 / R_{y}$, and zero boundary conditions. The linearized eigenvectors are

$$
\phi_{n m}=\sin n \pi x \sin m \pi y
$$

where $n, m \geqslant 1$. The corresponding eigenvalues are

$$
\lambda_{n m}=\frac{\left(n^{2}+m^{2}\right)^{4}+\left(k_{y} n^{2}+k_{x} m^{2}\right)^{2}}{\left(n^{2}+m^{2}\right)^{2}\left(\mathrm{~N}_{x} n^{2}+\mathrm{N}_{y} m^{2}\right)} .
$$




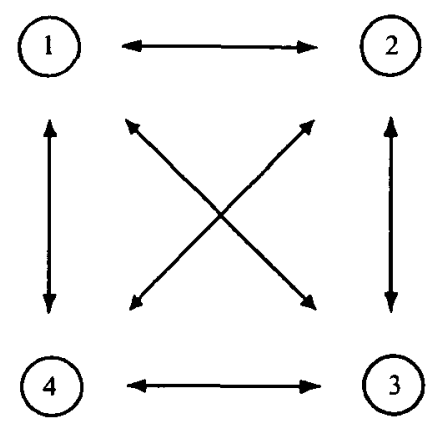

Fig. 7. Four-cell network.

If we want to be able to exchange $x$ and $y$ we must assume that $\mathrm{N}_{x}=\mathrm{N}_{y}=N$ and $k_{x}=k_{y}=k$. In that case

$$
\lambda_{n m}=\frac{\left(n^{2}+m^{2}\right)^{2}+k^{2}}{N\left(n^{2}+m^{2}\right)} \text {. }
$$

Note that when $\lambda_{n m}$, considered as a function on $\mathbb{N}^{2}$, achieves its minimum over the integers for a unique pair $n \neq m$, we have a two-dimensional kernel with the required $\mathbf{D}_{4}$-symmetry. We therefore expect the generic bifurcations in such circumstances to be described by the appropriate normal form derived above. A full nonlinear analysis, confirming this expectation, is in preparation.

\subsection{Four-cell rings}

Another different type of model with the same abstract structure is given by four interconnected cells as in Fig. 7. Here the model equations take the form

$$
\dot{x}=F^{\prime}(x, \lambda, \alpha),
$$

where $x \in \mathbb{R}^{4 n}, \lambda=\left(\lambda_{1}, \lambda_{2}\right) \in \mathbb{R}^{2}$ are the main bifurcation parameters and $\alpha \in \mathbb{R}^{k}$ represents unfolding parameters.

We consider the question of forced symmetry breaking as a parameter varies. We suppose first that $\lambda_{2}=0 ; F$ is a $\mathbf{D}_{4}$-equivariant problem which represents identical cells coupled such that all the near-neighbour interactions and all the diagonal interactions are the same.

The generators of $\mathbf{D}_{4}$ are $\alpha$, the mid-edge symmetry, represented by the permutation $(1,2,3,4) \mapsto(2,1,4,3)$, and $\beta$, the diagonal symmetry $(1,2,3,4) \mapsto(3,2,1,4)$. When $\lambda_{2}$ becomes non-zero we lose the $D_{4}$-symmetry. Generically, we get one of two representations of $\mathbf{Z}_{2} \oplus \mathbf{Z}_{2}$ or $\mathbf{Z}_{\mathbf{4}}$ :

1. (a) $Z_{2} \oplus Z_{2}$, generated by $\langle\alpha, \beta \alpha \beta\rangle$ (the symmetry of the rectangle),

(b) $Z_{2} \oplus Z_{2}$, generated by $\langle\beta, \alpha \beta \alpha\rangle$.

2. $Z_{4}$, generated by $\langle\alpha \beta\rangle$.

We assume here that symmetry-breaking occurs with the nearest-neighbour interactions equal only on opposite pairs of edges; that is, when $\lambda_{2} \neq 0$ we have the first situation $1 \mathrm{a}$. It is easy to see that the components of $F$ satisfy

$$
F\left(x_{1}, x_{2}, x_{3}, x_{4}, \lambda, \alpha\right)=\left(\begin{array}{l}
f\left(x_{1}, x_{2}, x_{3}, x_{4}, \lambda, \alpha\right) \\
f\left(x_{2}, x_{1}, x_{4}, x_{3}, \lambda, \alpha\right) \\
f\left(x_{3}, x_{4}, x_{1}, x_{2}, \lambda, \alpha\right) \\
f\left(x_{1}, x_{3}, x_{2}, x_{4}, \lambda, \alpha\right)
\end{array}\right)
$$


for some function $f$ with the following $(x, \lambda)$-combined symmetry:

$$
f\left(x_{1}, x_{2}, x_{3}, x_{4}, \lambda_{1}, \lambda_{2}\right)=f\left(x_{1}, x_{4}, x_{3}, x_{2}, \lambda_{1},-\lambda_{2}\right) .
$$

We introduce the change of variables on $\mathbb{R}^{4 n}$ given by the matrix

$$
T=\frac{1}{2}\left(\begin{array}{rrrr}
1 & 1 & 1 & -1 \\
1 & -1 & 1 & 1 \\
1 & 1 & -1 & 1 \\
1 & -1 & -1 & -1
\end{array}\right) \otimes I_{n}
$$

The new coordinates represent $X_{1} \sim(x, x, x, x), X_{2} \sim(x,-x, x,-x), X_{3} \sim$ $(x, x,-x,-x)$ and $X_{4} \sim(x,-x,-x, x)$. In those coordinates the linearization of

$$
\dot{X}=G(X, \lambda, \alpha)=T^{T} F(T X, \lambda, \alpha)
$$

at the origin $X=x=0$ is diagonal with elements $A+C+B+D, A+C-B-D$, $A-C+B-D$ and $A-C-B+D$, where $A=f_{x_{1}}^{o}, B=f_{x_{2}}^{o}, C=f_{x_{3}}^{o}$ and $D=f_{x_{4}}^{o}$ Moreover the symmetry (5.3) means that $A$ and $C$ are even in $\lambda_{2}$ and $D\left(\lambda_{2}\right)=B\left(-\lambda_{2}\right)$. Thus if $B$ and $D$ are also even in $\lambda_{2}$ there is no symmetry-breaking, so (5.1) is still $\mathbf{D}_{4}$. equivariant when $\lambda_{2} \neq 0$. On the other hand, when $B$ or $D$ have an odd component in $\lambda_{2}$, we do see symmetry-breaking.

When $\lambda_{2}=0$ we find that $B^{o}=D^{o}$, so that

$$
\sigma\left(D G^{o}\right)=\sigma\left(A^{o}+C^{o}+2 B^{o}\right) \cup \sigma\left(A^{o}+C^{o}-2 B^{o}\right) \cup \sigma\left(A^{o}-C^{o}\right) \text { (twice), }
$$

where $\sigma$ denotes the spectrum. Generically, we see that to get a two-dimensional kernel with the required $\mathbf{D}_{4}$-symmetry we must ensure that the trivial solution loses stability through a (double) eigenvalue of $A^{o}-C^{o}$ crossing the imaginary axis to the right. In the exceptional case when $B^{o}=D^{o}=0$, that is, when the linear nearneighbour interactions vanish, it is also possible to work with the eigenvalues of $A^{o}+C^{o}$.

The quantity $A^{0}-C^{o}$ represents the difference of the self-attraction and the diagonal interaction. In the original coordinates, the kernel of $D F^{o}$ represents steady states of the type $(u, v,-u,-v)$; that is, of diagonally opposite states. The exceptional case when $\sigma\left(A^{o}+C^{o}\right)$ changes sign corresponds to $(u, v, u, v)$, diagonally identical states.

\section{REFERENCES}

[1] T. J. Bridges and J. E. FURTER. Singularity theory and equivariant symplectic maps. Lecture Notes in Math. 1558 (Springer-Verlag, 1993).

[2] D. R. J. Chillingworth. Bifurcation from an orbit of symmetry. In Singularities and dynamical systems (ed. S. N. Pnevmatikos), 258-294 (North-Holland, 1986).

[3] J. Damov. The Unfolding and Determinacy Theorems for subgroups of $\mathscr{A}$ and $\mathscr{K}$. Memoirs of the American Mathematical Society 50 no. 306, 1984.

[4] J. E. Furter, A. M. SitTa and I. Stewart. Algebraic path formulation for equivariant bifurcation problems, preprint, 1995.

[5] T. GaffNey. Some new results in the classification theory of bifurcation problems. In Multiparameter bifurcation theory. Contemporary Mathematics 56 (Amer. Math. Soc., 1986).

[6] M. Golvbitsky and M. Roberts. A classification of degenerate Hopf bifurcations withO(2) symmetry. J. Diff. Eq. 69 (1987), 216-264.

[7] M. Golvbitsky and D. G. SChaefFer. A theory for imperfect bifurcation theory via singularity theory. Commun. Pure Appl. Wath. 32 (1979), 21-98. 


\section{Jacques-Élie Furter, Angela Maria Sitta and Ian Stewart}

[8] M. Golubitsky and D. G. SchaefFer. Singularities and groups in bifurcation theory, vol. I, Applied Math. Sci. 51 (Springer-Verlag, 1985).

[9] M. Goldbitsky, I. STEWART and D. G. SChAEFFER. Singularities and groups in bifurcation theory, vol. II, Applied Math. Sci. 69 (Springer-Verlag, 1988).

[10] A. LaRi-Lavassani. Multiparameter bifurcation with symmetry via singularity theory, Ph.D. Thesis (Ohio State U., 1990).

[11] D. Mond and J. Montaldi. Deformations of maps on complete intersections, Damon's $\mathscr{K}_{V^{-}}$ equivalence, and bifurcations, preprint, U. of Warwick, 1991.

[12] M. Peters. Classification of two-parameter bifurcations, Ph.D. Thesis, U. of Warwick, 1991.

[13] G. Schwartz. Smooth functions invariant under the action of a compact Lie group. Topology 14 (1975), 63-68.

[14] A. M. SiтTA. Singularity theory and equivariant bifurcation problems with parameter symmetry, $\mathrm{PhD}$ Thesis, U. of Warwick and USP-São Carlos, 1993.

[15] A. VANDERBaUwhede. Stability of bifurcating equilibria and the principle of reduced stability; in Bifurcation theory and applications, Montecatini 1983 (ed. L. Salvadori), Lectures Notes in Math. 1057 (Springer-Verlag, 1984), 209-233.

[16] A. VANDERBaUWHeDe. Local bifurcation and symmetry. Research Notes in Math. 75 (Pitman, 1982). 